\title{
The $(N=1)$ Supersymmetric Sine-Gordon Model in Two Dimensions. II
}

\author{
Marzio Cassandro ${ }^{1}$, Francesco Nicolò ${ }^{2}$, and Benedetto Scoppola ${ }^{3}$ \\ ${ }^{1}$ Dipartimento di Fisica, Università della Sapienza, C.N.R-G.N.S.M., C.N.R-G.N.F.M., Roma, \\ Italy \\ ${ }^{2}$ Dipartimento di Fisica, Università di Lecce, Lecce, Italy \\ ${ }^{3}$ I.N.F.N., Sezione di Roma, Roma, Italy
}

\begin{abstract}
In this second paper the technical part of the results about the Supersymmetric $N=1$ massless Sine-Gordon field theory, at finite (space) volume, is given. The proof that the theory exists and is analytic in the coupling constant $\lambda$ and that, at finite (space) volume, its Witten index is 1 , is, therefore, completed.
\end{abstract}

\section{Table of Contents}

Introduction . . . . . . . . . . . . . . . . . . . . . . . . . . . . 123

1. The Definition of the Effective Potential . . . . . . . . . . . . . . . . 124

2. The Tree Expansion . . . . . . . . . . . . . . . . . . . . . . . . 124

3. The Counterterms . . . . . . . . . . . . . . . . . . . . . . 125

4. The Recursive Construction of the Effective Potential. . . . . . . . . . . . 125

5. The Factorization Theorem . . . . . . . . . . . . . . . . . . . . . 127

6. The Bosonic Estimates . . . . . . . . . . . . . . . . . . . . . . . 130

7. The Fermionic Estimates . . . . . . . . . . . . . . . . . . . . . . 132

8. The Final Estimates of the Effective Potential I . . . . . . . . . . . . . . 133

9. The Final Estimates of the Effective Potential II. . . . . . . . . . . . . . 135

10. Conclusions. . . . . . . . . . . . . . . . . . . . . . . . . . . 142

\section{Introduction}

This paper completes the previous one [hereafter called (I)] giving a reasonably detailed version of the proof of Theorem 1 . We have chosen not to squeeze this proof in the appendix of (I) because it is another example (after those given in Gallavotti, Gallavotti and Nicolò [1] and Benfatto, Gallavotti and Nicolò [2]) of how the tree expansion allows us to use the Renormalization Group to provide very good estimates of the perturbative expansion of the field theories. 


\section{The Definition of the Effective Potential}

As discussed in Sect. (4)(I) the effective potential is defined by the equation

$$
e^{V_{N}^{(K)}}=\int \prod_{K+1}^{N} P\left(d \varphi^{(h)}\right) \prod_{K+1}^{N} \mu\left(d \psi^{(h)}\right) e^{V_{N}^{(N)}}
$$

Remark. All symbols we do not define here have been defined in (I) and their definition is unchanged.

The original Euclidean interaction $V^{(N)}\left(\varphi^{[\leqq N]}, \psi^{[\leqq N]}\right)$ defined in (3.3), (I) is rewritten in a slightly different way for technical purposes:

$$
\begin{aligned}
V^{(N)}= & \frac{-1}{2} \alpha^{2}\left\{-i \lambda \sum_{\sigma} \int_{\Lambda} d^{2} x \int d^{2} \theta: e^{i \alpha \sigma \varphi[N](x)}:: e^{i \theta \cdot \psi^{[\leqq N]}(x)}:+\frac{\lambda^{2}}{4} \sum_{\sigma_{1} \sigma_{2}} \sigma_{1} \sigma_{2}\right. \\
& \left.\times \int_{\Lambda^{2}} d^{2} \tilde{x} d^{2} \tilde{y}: e^{i \alpha\left(\sigma_{1} \varphi\left[N{ }^{[}(\tilde{x})+\sigma_{2} \varphi^{[} \leqq N\right](\tilde{y})\right)}: e^{-\alpha^{2} \sigma_{1} \sigma_{2} C[\leqq N](\tilde{x}, \tilde{y})} D^{[\leqq N]}(\tilde{x}, \tilde{y})\right\} \\
= & V_{1}^{(N)}+V_{2}^{(N)},
\end{aligned}
$$

where we used that $\psi^{(2)} \cdot \psi^{(1)}=-2 i \psi_{1}^{(1)} \psi_{2}^{(1)}$, the fact that $: e^{i \theta \cdot \psi^{[\leqq N]}(x)}:=e^{\left.i \theta \cdot \psi^{[} \leqq N\right](x)}$ as $S^{[\leqq N]}(0)=\left\langle\psi^{[\leqq N]}(x) \psi^{[\leqq N]}(x)\right\rangle=0$, and finally the well known relation:

$$
: e^{i \alpha \sigma_{1} \varphi(\tilde{x})}:: e^{i \alpha \sigma_{1} \varphi(\tilde{y})}:=: e^{i \alpha\left(\sigma_{1} \varphi(\tilde{x})+\sigma_{2} \varphi(\tilde{y})\right)}: e^{-\alpha^{2} \sigma_{1} \sigma_{2} C(\tilde{x}, \tilde{y})} .
$$

Remarks. i) The reintroduction of Grassmann variables $\theta$ 's to write

$$
\psi^{(2)} \cdot \psi^{(1)}=-2 i \int d^{2} \theta e^{i \theta \cdot \psi^{(1)}}
$$

is a useful technical device. It has nothing to do with the notion of superfield which

we will not use anymore.
ii) With $\Lambda$ we will always mean the space-time volume $\left[-\frac{T}{2}, \frac{T}{2}\right] \times\left[-\frac{L}{2}, \frac{L}{2}\right]$,
where $T$ can be finite or infinite. where $T$ can be finite or infinite.

\section{The Tree Expansion}

We will not give here the details of the tree expansion but we refer to Gallavotti et al. [1], and Pordt [3]; we just recall that one gets it starting from (1.4), (I) with the following recipes:

a) Integrate one frequency scale after the other,

b) At each frequency perform a cumulant expansion (in $\lambda$ ),

c) Collect this multiple expansion together.

A tree is just a graphical way of individuating a specific term of this expansion, which is made of truncated expectations of truncated expectations of ... on different scales. The point is that we can get a very good estimate for each of these terms which takes care of the natural length scales of the various factors.

Tree expansion allows us to incorporate pretty well the renormalization counterterms and this is its more relavant aspect so that one is also able to use it to study the flow of the running coupling constant.

In the present case, nevertheless the theory is relatively simple and does not need counterterms as discussed in the next section. Tree expansion is, here, just a reorganizing device apt to get best estimates. 


\section{The Counterterms}

As discussed in Sect. 9 of (I) in the scalar Sine-Gordon theory the only counterterms are constant counterterms (field independent) $:=C_{k}(\Lambda, N), k \leqq n$ if $\alpha^{2} \in\left[\alpha_{n-1}^{2}, \alpha_{n}^{2}\right)$ which are divergent as $N \rightarrow \infty$. No field dependent counterterms are needed up to $\alpha^{2}=8 \pi$, where the theory is not anymore superrenormalizable.

An analogous situation is present here with the difference that now

$$
\alpha_{n}^{2}=8 \pi\left(1-\frac{1}{2 n}\right)-4 \pi=4 \pi\left(1-\frac{1}{n}\right) .
$$

Nevertheless it is well known that the supersymmetry, has the effect of "smoothing" the divergences. This is what, of course, happens also here. In fact if the theory is regularized in the ultraviolet and in the infrared in such a way to be still supersymmetric in the sense discussed in Sect. 2 of (I) then it follows from the results of Sect. 6 that all the counterterms are equal to zero:

$$
C_{k}(\Lambda, N)=0 \quad \forall k, N, \Lambda .
$$

For technical reasons this has been proven up to $\alpha^{2}=2 \pi$, but we conjecture that it is true at least up to $\alpha^{2}=4 \pi$.

Moreover also if we would choose a regularization of the theory which does not preserve the supersymmetry, the original supersymmetry still leaves its mark in the sense that now the counterterms, one would introduce in analogy with the scalar case, are not zero, but remain finite as $N \rightarrow \infty$.

\section{The Recursive Construction of the Effective Potential}

As discussed in Sect. 2, see the references therein, a tree is a graphical object corresponding to different truncated expectations. At any bifurcation of a tree is associated a frequency $h$ corresponding to the scale length $\gamma^{-h}$ of the corresponding truncated expectation. Therefore in a tree expansion there will be a sum over the trees and a sum over the frequencies of the trees.

Starting from the definition (1.1) of the interaction it is possible to write the general structure of the expansion of the effective potential on scale $k$ :

where

$$
\begin{aligned}
& \tilde{V}^{(k)}=\sum_{1}^{\infty} n \sum_{\substack{0 \\
q_{1}+q_{2}=n}}^{n} \lambda^{q_{1}+2 q_{2}} \sum_{\sigma, \alpha_{1} ; \sigma_{2}} \int d^{2} \underline{\theta} \int_{A^{q_{1}}} d^{2} \underline{x} \int_{\Lambda^{2 q_{2}}} d^{2} \underline{\tilde{x}} d^{2} \underline{\underline{y}} \\
& : e^{i \alpha\left(\varphi[\leqq k](\underline{x}, \boldsymbol{\sigma})+\varphi[\leqq k]\left(\tilde{x}, \sigma_{1}\right)+\varphi[\leqq k]\left(\tilde{y}, \sigma_{2}\right)\right)}:: e^{i \varphi\left[\sum k\right](x, \theta)}: a_{n ; q_{1}}^{(k)}(\Lambda, N ; \underline{\underline{x}}, \underline{\underline{\sigma}}, \underline{\theta}),
\end{aligned}
$$

$$
\begin{aligned}
\varphi^{[\leqq k]}(\underline{x}, \underline{\sigma})=\sum_{i}^{b} \sigma_{i} \varphi^{[\leqq k]}\left(x_{i}\right), \\
\psi^{[\leqq k]}(\underline{x}, \underline{\theta})=\sum_{i}^{b}\left(\theta_{i} \cdot \psi^{[\leqq k]}\left(x_{i}\right)\right), \\
d^{2} \underline{\theta}=d^{2} \theta_{1} d^{2} \theta_{2} \ldots d^{2} \theta_{q_{1}}, \\
\underline{\underline{x}}=(\underline{x}, \underline{\tilde{x}}, \underline{\tilde{y}}), \\
\underline{\underline{\sigma}}=\left(\underline{\sigma}, \underline{\sigma}_{1}, \underline{\sigma}_{2}\right),
\end{aligned}
$$


and the Wick product is defined as usual.

The crucial part of the tree expansion is still hidden in the $a_{n ; q_{1}}^{(k)}(\Lambda, N ; \underline{\underline{x}}, \underline{\underline{\sigma}}, \underline{\theta})$. Its explicit expression is:

$$
a_{n ; q_{1}}^{(k)}(\Lambda, N ; \underline{\underline{x}}, \underline{\underline{\sigma}}, \underline{\theta})=\sum_{\substack{v_{1}(\tau)=q_{1} \\ v_{2}(\tau)=q_{2}}} \sum_{h_{0}>k}\left[\underline{\sigma}_{1} ; \underline{\sigma}_{2}\right] \prod_{1}^{q_{2}} D^{[\leqq N]}\left(\tilde{x}_{j}, \tilde{y}_{j}\right) V_{\Lambda, N}^{(k)}(\tau, \underline{h} ; \underline{\underline{x}}, \underline{\underline{\sigma}}, \underline{\theta}),
$$

where $\sum_{\tau}$ is the sum over the topological trees, $\sum_{h}$ is the sum over the frequencies of the tree bifurcations. $v_{1}(\tau)$ and $v_{2}(\tau)$ fix the number of final lines associated respectively to $V_{1}$ and $V_{2}, h_{0}$ is the frequency of the lowest bifurcation, $\left[\sigma_{1} ; \underline{\sigma}_{2}\right]$ is a product of $\sigma$ 's due to the decomposition of the $\sin \alpha \varphi$ functions appearing in $V_{2}$ and the $D^{[\leqq N]}$ also are associated to the final lines of $V_{2}$ type.

The whole problem to control the $a_{n ; q_{1}}^{(k)}$ is, of course, to have an explicit expression of $V_{A, N}^{(k)}(\tau, \underline{h} ; \underline{\underline{x}}, \underline{\underline{\sigma}}, \underline{\theta})$. Before starting the construction of $V_{A, N}^{(k)}(\tau, \underline{h} ; \underline{\underline{x}}, \underline{\underline{\sigma}}, \underline{\theta})$ with a recursive technique we rephrase the estimate of Theorem 1 of $(\mathrm{I})$ in terms of the $a_{n ; q_{1}}^{(k)}(\Lambda, N ; \underline{\underline{x}}, \underline{\underline{\sigma}}, \underline{\theta})$ in the following way (we leave the transcription to the reader):

Estimates of Theorem 1. We define

$$
\left\|a_{n ; q_{1}}^{(k)}(\Lambda, N ; \underline{\underline{x}}, \underline{\underline{\sigma}}, \underline{\theta}) \theta_{I}\right\|=\sum_{\underline{\underline{\sigma}}} \int_{\Lambda^{q_{1}}+2 q_{2}} d^{2} \underline{\underline{x}} \delta\left(x_{1}\right)\left|\int d^{2} \underline{\theta} \theta_{I} a_{n ; q_{1}}^{(k)}(\Lambda, N ; \underline{\underline{x}}, \underline{\underline{\sigma}}, \underline{\theta})\right|,
$$

where we excluded one integration using the translation invariance, and

$$
\theta_{I}=\prod_{j \in I} \theta_{j} \quad j=\left(\alpha_{i}, i\right) \quad \alpha_{i} \in(1,2) \quad i \in\left\{1, \ldots, q_{1}\right\} .
$$

Estimate (3.5) of (I) is equivalent to:

$$
\left\|a_{n ; q_{1}}^{(k)}(\Lambda, N ; \underline{\underline{x}}, \underline{\underline{\sigma}}, \underline{\theta}) \theta_{I}\right\| \leqq C^{\left(q_{1}+2 q_{2}\right)},
$$

where for $k \geqq 0 C$ depends on $\alpha$ but not on $k, \Lambda, N$.

For $k=-1$ (in this case $\widetilde{V}^{(-1)}=\log Z$ )

$$
\left\|a_{n ; q_{1}}^{(-1)}(\Lambda, N ; \underline{\underline{x}}, \underline{\underline{\sigma}}, \underline{\theta}) \theta_{I}\right\| \leqq(C(L))^{\left(q_{1}+2 q_{2}\right)}
$$

and $C(L)$ diverges as $L \rightarrow \infty$, but is still $N$ independent.

Remark. The factor $\theta_{I}$ tells which and how many $\psi^{[\leqq k]}(x)$ are present in the estimate of that part of the effective potential.

\subsection{Recursive Expression for $V_{A, N}^{(k)}$}

We assume that $\tau$ has $s_{0}$ subtrees $\tau_{1}, \ldots, \tau_{s_{0}}$ merging in its lowest bifurcation of frequency $h_{0}$. Graphically:

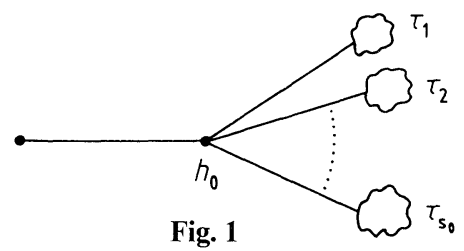


We consider the tree before integrating over the x's and summing over the $\sigma$ 's. Therefore any final line has a label $(x, \sigma, r)$, where $r \in\{1,2\}$ tells which potential $V_{r}$ is associated to that final branch. Fixed $(\underline{\underline{x}}, \underline{\underline{\sigma}}, \underline{r})$ we have the following relation:

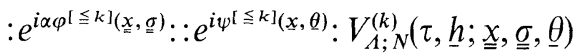

$$
\begin{aligned}
& =\frac{1}{s_{0} !} \prod_{1}^{s_{0}} V_{\lambda ; N}^{\left(h_{0}\right)}\left(\tau^{(i)}, \underline{h}^{(i)} ; \underline{\underline{x}}^{(i)}, \underline{\underline{G}}^{(i)}, \underline{\theta}^{(i)}\right) \mathscr{E}_{[>k]} \mathscr{E}_{\left[h_{0}\right]}^{T} \\
& \times\left(\left(: e^{i \alpha \varphi\left[h_{0} 1\left(\underline{\underline{x}}^{(1)}, \underline{\underline{\sigma}}^{(1)}\right)\right.}:: e^{i \psi\left[\leqq h_{0}\right]\left(\underline{x}^{(1)}, \theta^{(1)}\right)}:\right), \ldots\right. \\
& \left.\ldots,\left(: e^{i \alpha \varphi\left[\leqq h_{0}\right]\left(\underline{x}^{\left(s_{0}\right)}, \underline{g}^{\left(s_{0}\right)}\right)}:: e^{i \varphi\left[h_{0}\right]\left(\underline{x}^{\left(s_{0}\right)}, \theta^{\left(s_{0}\right)}\right)}:\right)\right),
\end{aligned}
$$

where

$$
\varphi^{[\leqq k]}(\underline{\underline{x}}, \underline{\underline{\sigma}})=\varphi^{[\leqq k]}(\underline{x}, \underline{\sigma})+\varphi^{[\leqq k]}\left(\underline{\tilde{x}}, \underline{\sigma}_{1}\right)+\varphi^{[\leqq k]}\left(\underline{\tilde{y}}, \underline{\sigma}_{2}\right),
$$

$\mathscr{E}_{[>k]}(\cdot)=\mathscr{E}_{\varphi[>k]}(\cdot) \mathscr{E}_{\psi[>k]}(\cdot)$ and the truncated expectation is defined with respect to the joint measure $\int P\left(d \varphi^{\left(h_{0}\right)}\right) \mu\left(d \psi^{\left(h_{0}\right)}\right)(\cdot)$.

Extracting from the right-hand side of (4.9) the dependence on the fields $\varphi^{[\leqq k]}$, $\psi^{[\leqq k]}$, with standard techniques we obtain the following recursion relation:

$$
\begin{aligned}
& V_{\Lambda, N}^{(k)}(\tau, \underline{h} ; \underline{\underline{x}}, \underline{\underline{\sigma}}, \underline{\theta})=\frac{1}{s_{0} !} \prod_{1}^{s_{0}} V_{\Lambda, N}^{\left(h_{0}\right)}\left(\tau^{(i)}, \underline{h}^{(i)} ; \underline{\underline{x}}^{(i)}, \underline{\underline{\sigma}}^{(i)}, \underline{\theta}^{(i)}\right) \\
& \times\left[\exp -\left(\frac{\alpha^{2}}{2}\left[U_{\varphi}^{\left[<h_{0}\right]}(\underline{\underline{x}}, \underline{\underline{\sigma}})-\sum_{1}^{s_{0}} U_{\varphi}^{\left[<h_{0}\right]}\left(\underline{\underline{x}}^{(i)}, \underline{\underline{\sigma}}^{(i)}\right)\right]\right)\right. \\
& \times \mathscr{E}_{\psi\left[<h_{0}\right]}\left(e^{i \psi\left[<h_{0}\right](x, \theta)}\right)\left(\prod_{1}^{s_{0}} \mathscr{E}_{\psi\left[<h_{0}\right]}\left(e^{\left.i \varphi^{\left.i<h_{0}\right]\left(x^{(i)}, \theta^{(i)}\right)}\right)}\right)^{-1}\right] \text {, } \\
& \mathscr{E}_{\left[h_{0}\right]}^{T}\left(\left(: e^{i \alpha \varphi\left[h_{0}\right]\left(\underline{x}^{(1)}, \underline{\sigma}^{(1)}\right)}:: e^{i \psi\left[h_{0}\right]\left(x^{(1)}, Q^{(1)}\right)}:\right), \ldots\right. \\
& \left.\ldots,\left(: e^{i \alpha \varphi\left[\leqq h_{0}\right]\left(\underline{x}^{\left(s_{0}\right)}, \underline{\sigma}^{\left(s_{0}\right)}\right)}:: e^{\left.i \varphi\left[\leqq h_{0}\right]\left(\underline{x}^{\left(s_{0}\right)}\right), \underline{\theta}^{\left(s_{0}\right)}\right)}:\right)\right)
\end{aligned}
$$

where:

$$
U_{\varphi}^{\left[<h_{0}\right]}(\tau)=U_{\varphi}^{\left[<h_{0}\right]}(\underline{\underline{x}}, \underline{\underline{\sigma}})=\sum_{\substack{i ; j j \\ i ; j \in \tau}} \sigma_{i} \sigma_{j} C^{\left[<h_{0}\right]}\left(x_{i}, x_{j}\right),
$$

and $x_{i}, x_{j}$ can be now $x, \tilde{x}, \tilde{y}$ and the same for $\sigma_{i}\left(\sigma_{j}\right)$.

From Eq. (4.10) one can write an explicit expression for $V_{\Lambda, N}^{(k)}$ but we will in fact look for a global estimate for this expression. To do that we have first to find an appropriate way of writing the factor

$$
\begin{aligned}
& \mathscr{E}_{\left[h_{0}\right]}^{T}\left(\left(: e^{i \alpha \varphi^{\left[h_{0}\right]}\left(\underline{x}^{(1)}, \underline{g}^{(1)}\right)}:: e^{i \psi^{\left[h_{0}\right]}\left(\underline{x}^{(1)}, \underline{Q}^{(1)}\right)}:\right), \ldots,\right.
\end{aligned}
$$

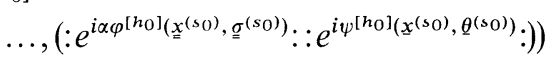

of (4.10). This will be discussed in the next section.

\section{The Factorization Theorem}

The results of this paper are mainly based on the possibility of obtaining an expression for $\frac{1}{s_{0} !} \mathscr{E}_{\left[h_{0}\right]}^{T}($ ) such that one can apply separately on it the bosonic and 
the fermionic estimates. To discuss this result, which does not strictly depend on this field theory model, we introduce some simplified notations.

We define

$$
\varphi(i)=\alpha \varphi^{\left[h_{0}\right]}\left(\underline{\underline{x}}^{(i)}, \underline{\underline{\sigma}}^{(i)}\right), \quad \psi(i)=i \psi^{\left[h_{0}\right]}\left(\underline{x}^{(i)}, \underline{\theta}^{(i)}\right) .
$$

Then

$$
\begin{aligned}
& \langle\varphi(i) \varphi(j)\rangle=-C(i, j)=\alpha^{2} U_{\varphi}^{\left[h_{0}\right]}\left(\tau^{(i)}, \tau^{l j)}\right), \\
& \langle\psi(i) \psi(j)\rangle=-S(i, j)=U_{\psi}^{\left[h_{0}\right]}\left(\tau^{(i)}, \tau^{(j)}\right),
\end{aligned}
$$

where

$$
\begin{aligned}
& U_{\varphi}^{\left[h_{0}\right]}\left(\tau^{(i)}, \tau^{(j)}\right)=\sum_{b \in \tau^{(i)} s \in \tau^{(j)}} \sigma_{b}^{(i)} \sigma_{s}^{(j)} C^{\left[h_{0}\right]}\left(x_{b}^{(i)}, x_{s}^{(j)}\right), \\
& U_{\psi}^{\left[h_{0}\right]}\left(\tau^{(i)}, \tau^{(j)}\right)=\sum_{b \in \tau^{(i)} s \in \tau^{(j)}} \theta_{b}^{(i)} \theta_{s}^{(j)} S^{\left[h_{0}\right]}\left(x_{b}^{(i)}, x_{s}^{(j)}\right) .
\end{aligned}
$$

We are interested to find an appropriate expression of

$$
\frac{1}{s !} \mathscr{E}^{T}\left(: e^{i(\varphi(1)+\psi(1))}:, \ldots,: e^{i(\varphi(s)+\psi(s))}:\right),
$$

where $\mathscr{E}^{T}$ is with respect to the product measure $\int P(d \varphi) \mu(d \psi)(\cdot)$.

Equation (5.4) can be expressed as a sum of Mayer graphs using only the algebraic structure of the truncated expectation, namely:

$$
(5.4)=\frac{1}{s !} \sum_{g \in G_{s}^{c}} \prod_{\{i ; j\} \in g}\left(e^{C(i, j)+S(i, j)}-1\right),
$$

where $G_{s}^{c}$ is the set of all the connected Mayer graph with vertices $\{1,2, \ldots, s\}$. The factorization theorem is the following (its proof is in Appendix A):

\section{Theorem 1.}

$$
\begin{aligned}
(5.4)= & \frac{1}{s !} \sum_{\left\{\boldsymbol{P}_{b}\right\}} \prod_{1}^{t}\left[\mathscr{E}\left(e^{i \psi\left(P_{b}\right)}\right) \prod_{j \in P_{b}} \mathscr{E}\left(e^{i \psi(j)}\right)^{-1}\right] \\
& \times \prod_{1}^{t} \mathscr{E}^{T}\left(: e^{i \varphi}:\left.\right|_{P_{b}}\right) \mathscr{E}^{T}\left(: e^{i \psi\left(P_{1}\right)}:, \ldots,: e^{i \psi\left(P_{t}\right)}:,: e^{i \psi\left(j_{1}\right)}:, \ldots,: e^{i \psi\left(j_{\tilde{s}}\right)}:\right) \\
= & \frac{1}{s !} \sum_{\left\{\boldsymbol{P}_{b}\right\}} \prod_{1}^{t} \mathscr{E}^{T}\left(: e^{i \varphi}:\left.\right|_{P_{b}}\right) \mathscr{E}^{T}\left(e^{i \psi\left(P_{1}\right)}, \ldots, e^{i \psi\left(P_{t}\right)}, e^{i \psi\left(j_{1}\right)}, \ldots, e^{i \psi\left(j_{\tilde{s}}\right)}\right) \\
& \times\left[\prod_{1}^{s} \mathscr{E}\left(e^{i \psi(i)}\right)^{-1}\right],
\end{aligned}
$$

where

and

$$
\left.\sum_{\left\{P_{b}\right\}}=\sum_{0}^{s} m \sum_{\substack{\{1 \\|\mathscr{R}|=m}} \sum_{\substack{s\} \\|\mathscr{R}|=m}}^{s} \sum_{\substack{\left(s_{1}, \ldots, s_{t}\right) \\ \Sigma_{b} s_{b}=m}} \sum_{\left|P_{b}\right|=s_{b}}^{\prime} P_{b}\right\}\left(\bigcup P_{b}=\mathscr{R}\right)
$$

$$
\left\{j_{1} \ldots j_{\tilde{s}}\right\}=\{1 \ldots s\}-\bigcup_{1}^{t} P_{b}
$$


Remarks. i) Fixed $m, \sum_{\mathscr{R}}$ is the sum over all the possible groups of $m$ vertices $\in\{1, \ldots, s\}$ and

$$
\frac{1}{s !} \sum_{\substack{\{1 \ldots \mathcal{R} \\|\mathscr{R}|=m}} 1=\frac{1}{m !(s-m) !}
$$

and we define $\tilde{s}=s-m$.

ii) For any fixed $\mathscr{R}$ and $t$ we remark that

$$
\sum_{\left(s_{1}, \ldots, s_{t}\right) ; \Sigma_{b} s_{b}=m} 1=C_{1}^{m}
$$

where $C_{1}$ is a constant independent from $\mathscr{R}$ and $t . \sum_{\left.\left|P_{b}\right|=P_{b}\right\}}$ is the sum over all the partitions of the vertices $\in \mathscr{R}$ in $t$ groups of $s_{1}, \ldots, s_{t}$ elements respectively. Moreover

iii)

$$
\begin{gathered}
\sum_{\left.\left|P_{b}\right|=s_{b}\right\}}^{\prime} 1=\left(\frac{m !}{s_{1} ! \ldots s_{t} !}\right) \frac{1}{t !} . \\
\psi\left(P_{b}\right)=\sum_{j \in P_{b}} \psi(j), \\
\mathscr{E}^{T}\left(: e^{i \varphi}:\left.\right|_{P_{b}}\right)=\mathscr{E}^{T}\left(: e^{i \varphi\left(a_{1}\right)}:, \ldots,: e^{i \varphi\left(a_{s_{b}}\right)}:\right),
\end{gathered}
$$

where $\mathscr{R} \supset P_{b}=\left\{a_{1}, \ldots, a_{s_{b}}\right\}$. that

iv) Finally we remark that, due to the definition of $\psi(i)$ in Eq. (5.1), it is clear

$$
\mathscr{E}\left(e^{i \psi(i)}\right)^{-1}=\mathscr{E}\left(e^{-i \psi(i)}\right)
$$

To understand how the estimates will be performed we rewrite (5.6) in the following way

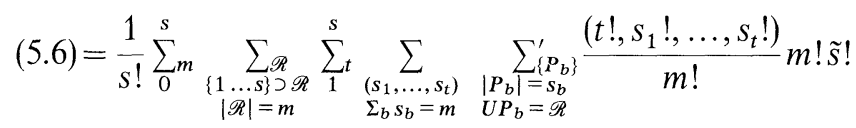

$$
\begin{aligned}
& \left\{\prod_{1}^{t} \frac{1}{s_{b} !} \mathscr{E}^{T}\left(: e^{i \varphi}:\left.\right|_{P_{b}}\right) \frac{1}{t ! \tilde{s} !} \mathscr{E}^{T}\left(e^{i \psi\left(\boldsymbol{P}_{1}\right)}, \ldots, e^{i \psi\left(\boldsymbol{P}_{t}\right)}, e^{i \psi\left(j_{1}\right)}, \ldots, e^{i \psi\left(j_{s}\right)}\right) \prod_{1}^{s} \mathscr{E}\left(e^{-i \psi(i)}\right)\right\}
\end{aligned}
$$

The claim is that, after integrating over the $\theta$ 's, we are able to give a good bound to the \{\} independent on the choice of $P_{b}$.

Once we pull the estimate of the \{\} part out of the multiple sum, from Eqs. (5.7)...(5.11) it follows:

$$
\frac{1}{s !} \sum_{0}^{s} \sum_{\substack{\{1 \ldots s\} \supset \mathscr{R} \\|\mathscr{R}|=m}} \sum_{1}^{s} t \sum_{\substack{\left(s_{1}, \ldots, s_{t}\right) \\ \Sigma_{b} s_{b}=m}} \sum_{\substack{\left.P_{b}\right\} \\\left\{P_{b}\right\}}}^{\prime}\left(t !, s_{1} !, \ldots, s_{t} !\right) \tilde{S} ! \leqq C_{2}^{s},
$$

where $C_{2}$ is a fixed number $>0$.

We insert (5.6) written as in (5.15) in (4.10) and we obtain a recursive expression for $V_{\Lambda, N}^{(k)}$. We are able to manage because we can provide appropriate estimates for 
all its parts,

$$
\begin{aligned}
& V_{\Lambda, N}^{(k)}(\tau, \underline{h} ; \underline{\underline{x}}, \underline{\underline{\sigma}}, \underline{\theta})=\frac{1}{s_{0} !} \sum_{0}^{s_{0}} m \sum_{\substack{\left\{1 \ldots S_{0} \mathcal{R} \supset \mathscr{R} \\
|\mathscr{R}|=m\right.}}^{\sum_{1}^{s_{0}} t \sum_{\substack{\left(s_{1}, \ldots, s_{t}\right) \\
\Sigma_{b} s_{b}=m}} \sum_{\left|P_{b}\right|=s_{b}}^{\prime}\left\{P_{b}\right\}_{v_{0}}} \tilde{s} ! \\
& \times\left(t !, s_{1} !, \ldots, s^{t} !\right)\left(\prod_{1}^{s_{0}} V_{\Lambda ; N}^{\left(h_{0}\right)}\left(\tau^{(i)}, \underline{h}^{(i)} ; \underline{\underline{x}}^{(i)}, \underline{\underline{\sigma}}^{(i)}, \underline{\theta}^{(i)}\right)\right. \\
& \left.\times\left[\exp -\left(\frac{\alpha^{2}}{2}\left[U_{\varphi}^{\left[<h_{0}\right]}(\underline{\underline{x}}, \underline{\underline{\sigma}})-\sum_{1}^{s_{0}} U_{\varphi}^{\left[<h_{0}\right]}\left(\underline{\underline{x}}^{(i)}, \underline{\underline{\sigma}}^{(i)}\right)\right]\right)\right]\right) \\
& \times\left[\mathscr{E}_{\psi\left[<h_{0}\right]}\left(e^{i \psi^{\left[<h_{0}\right]}(\underline{x}, \theta)}\right)\left(\prod_{1}^{s_{0}} \mathscr{E}_{\psi\left[<h_{0}\right]}\left(e^{i \psi^{\left[<h_{0}\right]}\left(\underline{x}^{(\imath)}, \theta^{(i)}\right)}\right)\right)^{-1}\right] \\
& \times\left(\prod _ { 1 } ^ { t } \frac { 1 } { S _ { b } ! } \mathscr { E } _ { ( h _ { 0 } ) } ^ { T } ( : e ^ { i \varphi ^ { ( h _ { 0 } ) } } : | _ { P _ { b } } ) \left[\frac { 1 } { t ! \tilde { S } ! } \mathscr { E } _ { ( h _ { 0 } ) } ^ { T } \left(e^{i \psi^{\left(h_{0}\right)}\left(P_{1}\right)}, \ldots,\right.\right.\right. \\
& \left.\left.\left.e^{i \psi^{\left(h_{0}\right)}\left(P_{t}\right)}, e^{i \psi^{\left(h_{0}\right)}\left(j_{1}\right)}, \ldots, e^{i \psi^{\left(h_{0}\right)}\left(j_{s}\right)}\right) \prod_{1}^{s_{0}} i \mathscr{E}_{\left(h_{0}\right)}\left(e^{-i \psi^{\left(h_{0}\right)}(i)}\right)\right]\right)
\end{aligned}
$$

and the $v_{0}$ means that we are referring to the $v_{h_{0}}$ bifurcation.

In the next section we prove the bosonic and the fermionic estimates which allow us to get an estimate for the coefficients of $V_{A, N}^{(k)}$ thought of as a polynomial in the $\theta$ 's variables.

\section{The Bosonic Estimates}

The bosonic estimate is an estimate for

$$
\begin{aligned}
& \mathscr{A}\left(\tau_{v}, \underline{h}_{v},\left\{P_{b}\right\}_{v}, \underline{\underline{x}}_{v}, \underline{\underline{\sigma}}_{v}\right) \\
& \quad=\left[\exp -\left(\frac{\alpha^{2}}{2}\left[U_{\varphi}^{\left[<h_{v}\right]}(\underline{\underline{x}}, \underline{\underline{\sigma}})-\sum_{1}^{s_{v}} U_{\varphi}^{\left[<h_{v}\right]}\left(\underline{\underline{x}}^{(i)}, \underline{\underline{\sigma}}^{(i)}\right)\right]\right)\right] \prod_{1}^{t} \frac{1}{s_{b} !} \mathscr{E}_{\left(h_{v}\right)}^{T}\left(: e^{i \varphi}:\left.\right|_{P_{b} ; v}\right) .
\end{aligned}
$$

We have introduced the subscript ${ }_{v}$ because this estimate will appear in many factors associated to different frequencies in the estimate of $V_{A ; N}^{(k)}$. Essentially, given a tree $\tau$, each of its bifurcations has a factor like (6.1). For the estimate being a useful one it must give:

a) a non-factorial dependence on the number $n_{v}$ of final lines merging in that bifurcation,

b) a good locality factor saying that this function is essentially local on the scale $\gamma^{-h_{v}}$

c) a good $h_{v}$ dependence which would make the final sum over the frequencies convergent as $N \rightarrow \infty$. We denote:

$$
\frac{1}{S_{b} !} \mathscr{E}_{\left(h_{v}\right)}^{T}\left(: e^{i \varphi}:\left.\right|_{P_{b} ; v}\right)=\frac{1}{S_{b} !} F^{\left(h_{v}\right)}\left(\tau_{v, P_{b}},\left.\underline{\underline{x}}_{v}\right|_{P_{b}},\left.\underline{\underline{\sigma}}_{v}\right|_{P_{b}}\right)
$$

where $\tau_{v}$ is the tree whose lowest bifurcation is $v$ and $P_{b}$ means the restrictions only to the subtrees associated to $P_{b}$. We estimate (6.2) using the Battle, Federbush technique [4], (see also Glimm et al. [5] and Gopfert and Mack [6]). 
The final result for a generic frequency $h_{v}$ and a generic tree $\tau$ is

$$
\begin{aligned}
& \left|\prod_{1}^{t} \frac{1}{S_{b} !} F^{\left(h_{v}\right)}\left(\tau_{v, P_{b}},\left.\underline{\underline{x}}_{v}\right|_{P_{b}},\left.\underline{\underline{\sigma}}_{v}\right|_{\boldsymbol{P}_{b}}\right)\right| \\
& \leqq\left(C_{3}(\gamma)\right)^{m-t} e^{\left.2 n_{v}\right|_{\mathscr{\rho}}} \prod_{1}^{t}\left[\operatorname { e x p } ( \frac { \alpha ^ { 2 } } { 2 } \sum _ { i \in P _ { b } } U _ { \varphi } ^ { [ h _ { v } ] } ( \underline { \underline { x } } ^ { ( i ) } , \underline { \underline { \sigma } } ^ { ( i ) } ) ) \operatorname { e x p } \left(-\delta \gamma^{h_{v}} d\left(\left.\tau_{v}\right|_{P_{b}}\right)\right.\right. \\
& \left.\times \prod_{i \in P_{b}}\left(\gamma^{h}\left|x_{\tau_{v}^{(i)}}\right|\right)^{(1-\varepsilon) \delta_{\tau_{\tau_{v}^{(i)}}}, 2 \delta_{Q} \underset{v}{(1)}, 0}\right] \text {, }
\end{aligned}
$$

where $C_{3}(\gamma)$ is a function of $\gamma$ which can be bounded by $\operatorname{Clog} \gamma$,

$$
\begin{aligned}
m=|\mathscr{P}|:= & \sum_{1}^{t}\left|P_{i}\right| \text { (remember that }\left\{P_{b}\right\} \text { is fixed), } \\
& \left.n_{v}\right|_{\mathscr{P}}=\left.\sum_{1}^{t} n_{v}\right|_{\mathscr{P}_{b}}=\sum_{1}^{t}{ }_{b} \sum_{i \in P_{b}} n_{v^{(i)}}
\end{aligned}
$$

and $n_{v^{(2)}}$ is the number of final lines which merge in the bifurcation $v^{(i)}$ which is one of the $s_{v}$ bifurcations immediately before $v$.

$\tau_{v}^{(i)}$ is the subtree of $\tau$ which has $v^{(i)}$ as lowest bifurcation.

$$
\left.U_{\varphi}^{\left[h_{\nu}\right]}\left(\underline{\underline{x}}^{(i)}, \underline{\underline{\sigma}}^{(i)}\right)=U_{\varphi}^{\left[h_{v}\right]}\left(\tau_{v}^{(i)}\right) \quad \text { [see Eq. }(4.10)\right] .
$$

$\delta$ is a number $\in(0,1)$,

$$
d\left(\left.\tau_{v}\right|_{P_{b}}\right)=d\left(\tau_{v^{\left(t_{1}\right)}}, \tau_{v^{\left(t_{2}\right)}}, \ldots, \tau_{\left.v^{\left(t_{s_{b}}\right.}\right)}\right)
$$

where $\left\{t_{1}, t_{2}, \ldots, t_{s_{b}}\right\}=P_{b}$ and $d\left(\tau_{1}, \ldots, \tau_{k}\right)$ is defined as the distance between the $k$ cluster associated to the subtrees $\tau_{1}, \ldots, \tau_{k} \cdot\left(\gamma^{h_{v}}\left|x_{\tau_{v}^{(i)}}\right|\right)$ is a slightly symbolic notation to indicate a first order zero appearing when all the points in $\tau_{v}^{(i)}$ shrink to a point. This zero turns out to be crucial in the estimates only when the subtrees $\tau_{v}^{(i)}$ has only two final lines and it is neutral. In that case $\left(\gamma^{h_{v}}\left|x_{\tau_{v}^{(i)}}\right|\right)=\left(\gamma^{h_{v}}\left|x^{+}-x^{-}\right|\right)$.

Therefore we have for the bosonic part the following bound:

$$
\begin{aligned}
&\left|\mathscr{A}\left(\tau_{v}, \underline{h}_{v},\left\{P_{b}\right\}_{v}, \underline{\underline{x}}_{v}, \underline{\underline{\sigma}}_{v}\right)\right| \\
& \leqq \exp \left[-\frac{\alpha^{2}}{2}\left[U_{\varphi}^{\left[<h_{v}\right]}\left(\tau_{v}\right)-\sum_{i}^{s_{v}}\left(U_{\varphi}^{\left[<h_{v}\right]}\left(\tau_{v}^{(i)}\right)+U_{\varphi}^{\left[h_{v}\right]}\left(\tau_{v}^{(i)}\right) \chi\left(i \in \cup_{b} P_{b}\right)\right)\right]\right] \\
& \times\left(C_{3}(\gamma)\right)^{m_{v}-t_{v}} e^{2 n_{v} \mid \mathscr{P}_{v}} \prod_{1}^{t_{v}} \exp \left(-\delta \gamma^{h_{v}} d\left(\left.\tau_{v}\right|_{P_{b}}\right)\right. \\
& \times \prod_{i \in P_{b}}\left(\gamma^{h_{v}}\left|x_{\tau_{v}^{(i)}}\right|\right)^{(1-\varepsilon) \delta_{n_{\tau_{v}^{(i)}}}, 2 \delta_{Q_{\tau_{v}}^{(i)}}, 0} .
\end{aligned}
$$




\section{The Fermionic Estimates}

From Eq. (5.17) we define:

$$
\begin{aligned}
\mathscr{B}\left(\tau_{v}, \underline{h}_{v},\left\{P_{1}\right\}_{v}, \underline{x}_{v}, \underline{\sigma}_{v}, \underline{\theta}^{(v)}\right) \\
=\left[\mathscr{E}_{\psi\left[<h_{v}\right]}\left(e^{i \psi\left[<h_{v}\right](\underline{x}, \theta)}\right)\left(\prod_{1}^{s_{v}} \mathscr{E}_{\psi\left[<h_{v}\right]}\left(e^{i \psi^{\left[<h_{v}\right]}\left(x^{(i)}, \theta^{(i)}\right)}\right)\right)^{-1}\right] \\
\quad \times\left[\frac{1}{t ! \tilde{s} !} \mathscr{E}_{\left(h_{v}\right)}^{T}\left(e^{i \psi^{\left(h_{v}\right)\left(P_{1}\right)}}, \ldots, e^{i \psi^{\left(h_{v}\right)}\left(P_{t}\right)}, e^{i \psi^{\left(h_{v}\right)\left(j_{1}\right)}}, \ldots, e^{i \psi^{\left(h_{v}\right)}\left(j_{s}\right)}\right) \prod_{1}^{s_{v}} \mathscr{E}_{\left(h_{v}\right)}\left(e^{\left.-i \psi^{\left(h_{v}\right)(i)}\right)}\right]\right.
\end{aligned}
$$

where $\underline{\theta}^{(v)}$ are the $\theta$ 's associated to the final lines of type $V_{1}$ [see Eq. (1.1)] which merge into the bifurcation $v$.

If $\tau$ has $q_{1}$ final lines in its bifurcation $v$, the number of $\theta$ 's which are present is at most $2\left(\left.q_{1}\right|_{v}\right) \leqq 2 n_{v}$, where $\left.q_{1}\right|_{v}$ is the number of lines of type 1 which merge in $v$. Therefore $\underline{\theta}^{(\bar{v})}$ is a $\left.2 q_{1}\right|_{v}$-components vector.

To get the fermionic estimates we have to expand $\mathscr{B}$,

where

$$
\mathscr{B}\left(\tau_{v}, \underline{h}_{v},\left\{P_{b}\right\}_{v}, \underline{x}_{v}, \underline{\sigma}_{v}, \underline{\theta}^{(v)}\right)=\sum_{\left.\left\{1 \ldots 2 q_{1}\right\}\right|_{v} \supset I_{v}} \theta_{I_{v}}^{(v)} B_{I_{v}}\left(\tau_{v}, \underline{h}_{v},\left\{P_{b}\right\}_{v}, \underline{x}_{v}, \sigma_{v}\right)
$$

$$
\theta_{I_{v}}^{(v)}=\prod_{j \in I_{v}} \theta_{j} .
$$

Remark. Looking at (7.1) it is clear that fixed $\theta_{I_{v}}^{(v)}, B_{I_{v}}$ is just the sum of the different terms in the expansion of (7.1) which produce a factor $\theta_{I_{v}}^{(v)}$ in front. The number of these terms is bounded.

In fact looking at (7.1) it is clear that there are $C_{4}^{\left|I_{v}\right|}$ terms, with $C_{4}>0$ independent from the other parameters. Moreover the following relation is trivial:

$$
\sum_{\left\{\left.1 \ldots 2 q_{1}\right|_{v} \supset I_{v}\right.} 1 \leqq C_{5}^{2\left(\left.q_{1}\right|_{v}\right)} \leqq C_{5}^{2 n_{v}} .
$$

Once that we know that $B_{I_{v}}$ of Eq. (7.2) is the sum of a controllable number of terms we look for an estimate of the generic of them. The generic addend of $B_{I_{v}}$ has exactly the form (7.1) with the only difference that each term $e^{i \psi}$ has to be replaced by a product of $\psi$ 's. It is on these expectation or truncated expectation of products of fermionic fields that we apply the Gawedzki-Kupiainen estimates they used to study the Gross-Neveu model [7].

Therefore we have

$$
B_{I_{v}}=\sum_{\varrho} B_{I_{v}}^{(\varrho)}
$$

where $\sum_{\varrho} 1=C_{4}^{\left|I_{v}\right|}$, and $B_{I_{v}}^{(\varrho)}$ satisfies the following $\varrho$-independent estimate:

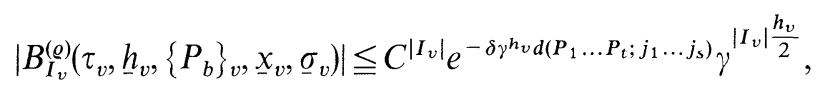

where $d\left(P_{1} \ldots P_{t} ; j_{1} \ldots j_{\tilde{s}}\right)$ is the distance between the subgroups of clusters $\left.\tau_{v}\right|_{P_{1}}, \ldots,\left.\tau_{v}\right|_{P_{t}}\left[\right.$ see Eq. (6.3)] and the clusters $\left.\tau_{v}\right|_{j_{1}}, \ldots,\left.\tau_{v}\right|_{j_{s}}$. The factor $\gamma^{\left|I_{v}\right| \frac{h_{v}}{2}}$ appears as each $\psi^{\left(h_{v}\right)}$ or $\psi^{\left[<h_{v}\right]}$ brings a factor $\gamma^{\frac{h_{v}}{2}}$. 


\section{The Final Estimates of the Effective Potential (I)}

From the definition (6.1), (7.1), and Eq. (5.17) we have:

$$
\begin{aligned}
& V_{A ; N}^{(k)}(\tau, \underline{h} ; \underline{\underline{x}}, \underline{\underline{\sigma}}, \underline{\theta}) \\
& \quad=\prod_{v \in \tau}\left\{\sum_{\left\{P_{b}\right\}_{v}} \mathscr{A}\left(\tau_{v}, \underline{h}_{v},\left\{P_{b}\right\}_{v}, \underline{x}_{v}, \underline{\underline{\sigma}}_{v}\right) \mathscr{B}\left(\tau_{v}, \underline{h}_{v},\left\{P_{b}\right\}_{v}, \underline{x}_{v}, \underline{\sigma}_{v}, \underline{\theta}^{(v)}\right)\right\}
\end{aligned}
$$

and

$$
\mathscr{B}\left(\tau_{v}, \underline{h}_{v},\left\{P_{b}\right\}_{v}, \underline{x}_{v}, \underline{\sigma}_{v}, \theta_{I_{v}}^{(v)}\right)=\sum_{\left.\left\{1 \ldots 2 q_{1}\right\}\right\}_{v} \supset I_{v}} \theta_{I_{v}}^{(v)} B_{I_{v}}\left(\tau_{v}, \underline{h}_{v},\left\{P_{b}\right\}_{v}, \underline{x}_{v}, \underline{\sigma}_{v}\right) .
$$

We need the following decomposition of $V_{\Lambda_{i}}^{(k)}(\tau, \underline{h} ; \underline{\underline{x}}, \underline{\underline{\sigma}}, \underline{\theta})$ :

$$
V_{\Lambda ; N}^{(k)}(\tau, \underline{h} ; \underline{\underline{x}}, \underline{\underline{\sigma}}, \underline{\theta})=\sum_{\left\{1 \ldots 2 q_{1}\right\} \supset I} \theta_{I} V_{\Lambda ; N ; I}^{(k)}(\tau, \underline{h} ; \underline{\underline{x}}, \underline{\underline{\sigma}}),
$$

where fixed $\tau,\left\{1 \ldots 2 q_{1}\right\}$ are the indices associated to the $\theta$ 's appearing on the final branches.

We observe now that we have the following identity:

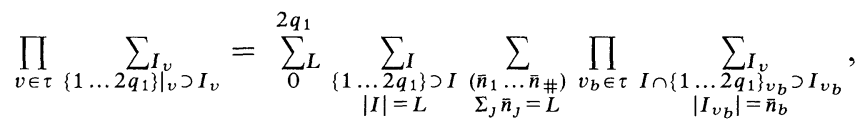

where $\tau$ is fixed and has in its final lines $2 q_{1} \theta$ 's. $L$ is just the number of the $\theta$ 's which are extracted: the order of the generic term of the polynomial (8.2).

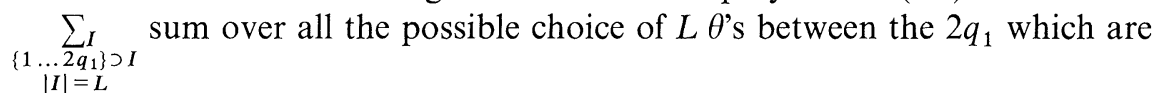
present. Moreover

$$
\sum_{0}^{2 q_{1}} L \underset{\substack{\left\{\ldots 2 q_{1}\right\} \supset I \\|I| \mid=L}}{\sum_{I}} 1 \leqq C_{5}^{2 q_{1}}
$$

\# is the number of bifurcation of $\tau$ which can have $\theta$ 's in their associated truncated expectations,

$$
\#=C_{6} q_{1} .
$$

$\sum_{\substack{\left(\bar{n}_{1} \ldots \bar{n}_{\#) \supset I} \\ \Sigma_{j} \bar{n}_{j}=L\right.}}$ chooses how many $\theta$ 's one picks from each of these bifurcations,

$$
\sum_{\substack{\left(\bar{n}_{1}, \ldots \bar{n} \#\right) \\ \Sigma, \tilde{n}_{j}=L}} 1 \leqq C_{7}^{L} \leqq C_{7}^{2 q_{1}}
$$

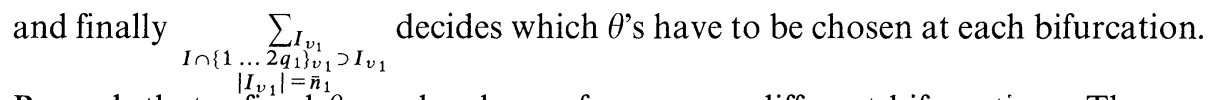
Remark that a fixed $\theta$ can be chosen from many different bifurcations. Then

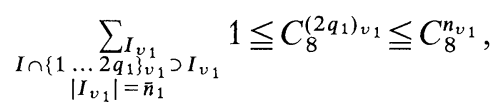


where $n_{v_{1}}$ are the final lines of type 1 which merge in the bifurcation $v_{1}$. Therefore we have

$$
\prod_{\left.v \in \tau\left\{1 \ldots 2 q_{1}\right\}\right|_{v} \supset I_{v}} \sum_{\left\{1 \ldots 2 q_{1}\right\} \supset I}\left(\prod_{v \in \tau} \sum_{I_{v} I_{v}=I}\right)
$$

and from the previous inequalities

$$
\prod_{v \in \tau} \sum_{\cup_{v} I_{v}=I} \leqq C_{7}^{2 q_{1}} \prod_{v \in \tau} C_{8}^{n_{v}}
$$

Therefore we can rewrite (8.2) in the following way:

$$
\begin{aligned}
& V_{\Lambda, N}^{(k)}(\tau, \underline{h} ; \underline{\underline{x}}, \underline{\underline{\sigma}}, \underline{\theta})=\sum_{\left\{1 \ldots 2 q_{1}\right\} \supset I} \theta_{I}\left(\prod_{v \in \tau} \sum_{\left\{P_{b}\right\}_{v}} \sum_{\cup_{v} I_{v}=I} I_{v}\left(\prod_{v} C_{8}^{n_{v}}\right)^{-1}\right) \\
& \quad \times\left[\prod_{v \in \tau} C_{2}^{s_{v}} C_{8}^{n_{v}} \mathscr{A}\left(\tau_{v}, \underline{h}_{v},\left\{P_{b}\right\}_{v}, \underline{x}_{v}, \underline{\underline{\sigma}}_{v}\right) B_{I_{v}}\left(\tau_{v}, \underline{h}_{v},\left\{P_{b}\right\}_{v}, \underline{x}_{v}, \underline{\sigma}_{v}\right)\right] .
\end{aligned}
$$

This is the appropriate expression to estimate. In fact we estimate the [ ] part independently from $\left\{P_{1}\right\}_{v}$ and $I_{v}$ and then, using (8.9), (5.16) and the definition

$$
\sum_{\left\{P_{b}\right\}_{v}}=\sum_{\left\{P_{b}\right\}_{v}} \tilde{s}_{v} !\left(t_{v} !, s_{1} !, \ldots, s_{t_{v}} !\right) C_{2}^{-s_{v}} \leqq 1
$$

one realizes that

$$
\left(\prod_{v \in \tau} \sum_{\left\{P_{b}\right\}_{v}} \sum_{\cup_{v} I_{v}=I}\left(\prod_{v} C_{8}^{n_{v}}\right)^{-1}\right) \leqq 1
$$

We conclude this section writing the explicit expression of the $a_{n ; q_{1}}^{(k)}(\Lambda, N ; \underline{\underline{x}}, \underline{\underline{\sigma}}, \underline{\theta})$ as an expression in the $\theta$ 's variables in terms of the quantities we will be able to estimate

$$
\begin{aligned}
& a_{n ; q_{1}}^{(k)}(\Lambda, N ; \underline{\underline{x}}, \underline{\underline{\sigma}}, \underline{\theta}) \\
& =\sum_{\left\{1 \ldots 2 q_{1}\right\} \supset I} \theta_{I}\left\{\sum_{\substack{\sum_{\tau} \\
v_{1}(\tau)=q_{1} \\
v_{2}(\tau)=q_{2}}} \sum_{h_{0}>k}\left[\underline{\sigma}_{1}: \underline{\sigma}_{2}\right]\right. \\
& \times\left(\prod_{v \in \tau} \sum_{\left\{P_{b}\right\}_{v}} \sum_{\cup_{v} I_{v}=I} I_{v}\left(\prod_{v} C_{8}^{n_{v}}\right)^{-1}\right)\left[\prod_{1}^{q_{2}} D^{[\leqq N]}\left(\tilde{x}_{j}, \tilde{y}_{j}\right)\right. \\
& \left.\left.\quad \times \prod_{v \in \tau} C_{2}^{s_{v}} C_{8}^{n_{v}} \mathscr{A}\left(\tau_{v}, \underline{h}_{v},\left\{P_{b}\right\}_{v}, \underline{\underline{x}}_{v}, \underline{\sigma}_{v}\right) B_{I_{v}}\left(\tau_{v}, \underline{h}_{v},\left\{P_{b}\right\}_{v}, \underline{x}_{v}, \underline{\sigma}_{v}\right)\right]\right\} \\
& =\sum_{\left\{1 \ldots 2 q_{1}\right\} \supset I} \theta_{I} a_{n ; q_{1} ; I}^{(k)}(\Lambda, N ; \underline{\underline{x}}, \underline{\underline{\sigma}}),
\end{aligned}
$$

and immediately:

$$
\begin{aligned}
& \left\|a_{n ; q_{1}}^{(k)}(\Lambda, N ; \underline{\underline{x}}, \underline{\underline{\sigma}}, \underline{\theta}) \theta_{I}\right\| \\
& \quad=\sum_{\underline{\underline{\sigma}}} \int_{\Lambda^{q_{1}}} d^{2} \underline{x} \int_{\Lambda^{2 q_{2}}} d^{2} \underline{\tilde{x}} d^{2} \underline{\underline{y}} \delta\left(x_{1}\right) \mid a_{n ; q_{1} ; I^{c}}^{(k)}(\Lambda, N ; \underline{\underline{x}}, \underline{\underline{\sigma}})\|=\| a_{n ; q_{1} ; I^{c}}^{(k)} \| .
\end{aligned}
$$

The study of the right-hand side of (8.14) is now accessible and it will be discussed in the next section. 


\section{The Final Estimate of the Effective Potential (II)}

From Eq. (8.13) of the previous section, it is clear that we need an explicit expression and an explicit estimate of

$$
\begin{aligned}
& \Gamma\left(\tau,\left\{\underline{h}_{v}\right\},\left\{\left\{P_{b}\right\}_{v}\right\},\left\{I_{v}\right\}, \underline{\underline{x}}, \underline{\underline{\sigma}}\right) \\
& \quad:=\left[\prod_{1}^{q_{2}} D^{\Gamma \leqq N]}\left(\tilde{x}_{j}, \tilde{y}_{j}\right) \prod_{v \in \tau} C_{2}^{s_{v}} C_{8}^{n_{v}} \mathscr{A}\left(\tau_{v}, \underline{h}_{v},\left\{P_{b}\right\}_{v}, \underline{\underline{x}}_{v}, \underline{\underline{\sigma}}_{v}\right) B_{I_{v}}\left(\tau_{v}, \underline{h}_{v},\left\{P_{b}\right\}_{v}, \underline{x}_{v}, \underline{\sigma}_{v}\right)\right]
\end{aligned}
$$

and of

$$
\int d \underline{\underline{x}} \delta\left(x_{1}\right)\left|\Gamma\left(\tau,\left\{\underline{h}_{v}\right\},\left\{\left\{P_{b}\right\}_{v}\right\},\left\{I_{v}\right\}, \underline{\underline{x}}, \underline{\underline{\sigma}}\right)\right| .
$$

We start considering the case in which $q_{2}=0$; this means that all the final branches of the tree $\tau$ bring the part $V_{1}^{(N)}$ of the interaction [see Eq. (1.1)]. In this case $n=v(\tau)=q_{1}$. From the estimates (7.5) and (6.4) we have:

$$
\begin{aligned}
& \left|\Gamma\left(\tau,\left\{\underline{h}_{v}\right\},\left\{\left\{P_{b}\right\}_{v}\right\},\left\{I_{v}\right\}, \underline{\underline{x}}, \underline{\underline{\sigma}}\right)\right| \\
& \leqq\left(\prod_{v \in \tau} \exp -\frac{\alpha^{2}}{2}\left[U_{\varphi}^{\left[<h_{v}\right]}\left(\tau_{v}\right)-U_{\varphi}^{\left[<h_{v^{\prime}}\right]}\left(\tau_{v}\right)-U_{\varphi}^{\left[h_{v^{\prime}}\right]}\left(\tau_{v}\right) \chi\left(v \in \mathscr{P}_{v^{\prime}}\right)\right]\right) \\
& \times\left(\prod_{1}^{q_{1}=n} \gamma^{\frac{\alpha^{2}}{4 \pi} h_{i}}\right) \prod_{v \in \tau}\left(\left(C_{3}(\gamma)\right)^{n_{v}-t_{v}} e^{2 n_{v}}\right)
\end{aligned}
$$

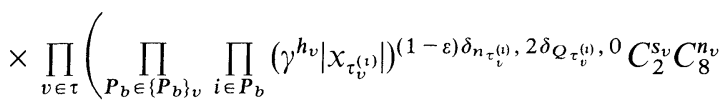

$$
\begin{aligned}
& \times \prod_{1}^{t_{v}} e\left(-\delta \gamma^{h_{v}} d\left(\left.\tau_{v}\right|_{P_{b}}\right) C^{\left|I_{v}\right|} \exp -\delta \gamma^{h_{v}} d\left(P_{1} \ldots P_{t_{v}} ; j_{1} \ldots j_{\tilde{s}_{v}}\right) \gamma^{\left|I_{v}\right| \frac{h_{v}}{2}}\right) .
\end{aligned}
$$

We remark that

i) The frequencies $h_{i}$ in the factor $\prod_{1}^{q_{1}=n} \gamma^{\frac{\alpha^{2}}{4 \pi}} h_{i}$ are the frequencies of the first bifurcations the final lines meet. $\gamma^{\frac{\alpha^{2}}{4 \pi} h}$ is, essentially, $\left.e^{\frac{\alpha^{2}}{2}} C l \leqq h\right]_{(0)}$.

ii)

$$
\sum_{1}^{t_{v}} d\left(\left.\tau_{v}\right|_{P_{b}}\right)+d\left(P_{1} \ldots P_{t_{v}} ; j_{1} \ldots j_{\tilde{s}_{v}}\right)=d^{*}\left(\tau_{v}\right),
$$

where $d^{*}\left(\tau_{v}\right)$ is the distance between the $s_{v}$ clusters merging in $\tau_{v}=$ length of the shortest path connecting all the clusters $\tau_{v_{1}}, \ldots, \tau_{v_{s_{v}}}$.

iii) $m_{v}-t_{v} \leqq s_{v}$ [for the definition of $m_{v}$ see Sect. 6, following Eq. (6.3)] We observe that for a generic choice $\left\{I_{v}\right\}$ with the constraint $\sum_{v}\left|I_{v}\right|=L \leqq 2 n_{v}$ we
have:

$$
\prod_{v \in \tau} \gamma^{\left|I_{v}\right| \frac{h_{v}}{2}}=\prod_{v \in \tau} \gamma^{\bar{n}_{v} \frac{h_{v}}{2}} \leqq \prod_{v \in \tau} \gamma^{\left(h_{v}-h_{v^{\prime}}\right)} \frac{\varrho_{v}}{2}
$$

with $\varrho_{v} \leqq 2 n_{v}, \bar{n}_{v}$ is the number of the $\theta$ 's which are chosen at $v$. As a $\theta$ cannot be chosen more than once $\varrho_{v^{\prime}}=\bar{n}_{v}+\bar{n}_{v^{\prime}} \leqq$ the number of $\theta$ 's which are in $\cup_{v} I_{v}$ and merge in $v^{\prime}$. 
We can rewrite $(9.3)$ as

$$
\begin{aligned}
& \left|\Gamma\left(\tau,\left\{\underline{h}_{v}\right\},\left\{\left\{P_{b}\right\}_{v}\right\},\left\{I_{v}\right\}, \underline{\underline{x}}, \underline{\underline{\sigma}}\right)\right| \\
& \leqq\left(\prod_{v \in \tau} \exp -\frac{\alpha^{2}}{2}\left[U_{\varphi}^{\left[<h_{v}\right]}\left(\tau_{v}\right)-U_{\varphi}^{\left[<h_{v^{\prime}}\right]}\left(\tau_{v}\right)-U_{\varphi}^{\left[h_{v^{\prime}}\right]}\left(\tau_{v}\right) \chi\left(v \in \mathscr{P}_{v^{\prime}}\right)\right]\right)\left(\begin{array}{c}
q_{1}=n \\
\prod_{1} i \gamma^{\frac{\alpha^{2}}{4 \pi}} h_{i}
\end{array}\right)
\end{aligned}
$$

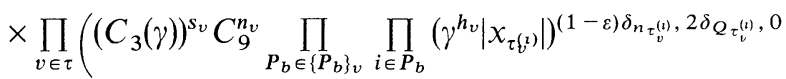

$$
\begin{aligned}
& \left.\times \exp \left(-\delta \gamma^{h_{v}} d^{*}\left(\tau_{v}\right)\right) \gamma^{\left(h_{v}-h_{v^{\prime}}\right) n_{v}}\right)
\end{aligned}
$$

In Appendix B we prove the following estimate for the first factor of (9.7),

$$
\begin{aligned}
& \left(\prod_{1}^{q_{1}=n} \gamma^{\frac{\alpha^{2}}{4 \pi} h_{\imath}}\right) \prod_{v \in \tau}\left(\exp -\frac{\alpha^{2}}{2}\left[U_{\varphi}^{\left[<h_{v}\right]}\left(\tau_{v}\right)-U_{\varphi}^{\left[<h_{v^{\prime}}\right]}\left(\tau_{v}\right)-U_{\varphi}^{\left[h_{v^{\prime}}\right]}\left(\tau_{v}\right) \chi\left(v \in \mathscr{P}_{v^{\prime}}\right)\right]\right. \\
& \left.\quad \times \exp \left(-\delta \gamma^{h_{v}} d^{*}\left(\tau_{v}\right)\right)\right) \leqq(C(M, \gamma))^{n}\left(\gamma^{\frac{\alpha^{2}}{4 \pi} k}\right)^{n} \\
& \quad \times \prod_{v \in \tau}\left(\gamma^{\frac{\alpha^{2}}{4 \pi} n_{v}\left(h_{v}-h_{v^{\prime}}\right)} \exp \left(-\tilde{\delta} \gamma^{h_{v}} d\left(\tau_{v}\right)\right)\right) \gamma^{-\frac{\alpha^{2}}{4 \pi} Q_{v}^{2}\left(h_{v}-\left(h_{v^{\prime}}+1\right)\right)},
\end{aligned}
$$

where $M$ is a fixed number defined in Appendix B.

Plugging this estimate in (9.7) we get:

$$
\begin{aligned}
&\left|\Gamma\left(\tau,\left\{\underline{h}_{v}\right\},\left\{\left\{P_{b}\right\}_{v}\right\},\left\{I_{v}\right\}, \underline{\underline{x}}, \underline{\underline{\sigma}}\right)\right| \\
& \leqq(C(M, \gamma))^{n}\left(\gamma^{\frac{\alpha^{2}}{4 \pi} k}\right)^{n} \prod_{v \in \tau}\left(\gamma^{\frac{\alpha^{2}}{4 \pi} n_{v}\left(h_{v}-h_{v^{\prime}}\right)} \gamma^{n_{v}\left(h_{v}-h_{v^{\prime}}\right)}\left(C_{3}(\gamma)\right)^{s_{v}} C_{9}^{n_{v}}\right. \\
&\left.\times \prod_{P_{b} \in\left\{P_{b}\right\}_{v}} \prod_{i \in P_{b}}\left(\gamma^{h_{v}}\left|x_{\tau_{v}^{(i)}}\right|\right)^{(1-\varepsilon) \delta_{\tau_{\tau_{v}}^{(i)}}, 2 \delta_{Q_{\tau_{v}}^{(i)}}, 0} \exp \left(-\tilde{\delta} \gamma^{h_{v}} d\left(\tau_{v}\right)\right)\right) .
\end{aligned}
$$

Remark. The $\delta_{n \tau \tau^{(2)}, 2}$ is introduced just because we will not make any use of these zeroes when $n_{v_{t}}>2$. This will be discussed later on. Using the obvious inequality:

$$
\exp \left(-\widetilde{\delta} \gamma^{h_{v}} d\left(x_{1}, x_{2}\right)\right) \gamma^{h_{v^{\prime}}}\left|x_{1}-x_{2}\right| \leqq(\mathrm{const}) \gamma^{-\left(h_{v}-h_{v^{\prime}}\right)} \exp \left(-\tilde{\delta}^{\prime} \gamma^{h_{v}} d\left(x_{1}, x_{2}\right)\right)(9.10)
$$

with $\widetilde{\delta}^{\prime}<\tilde{\delta}$, we easily get (see Gallavotti [8, Appendix A]),

$$
\begin{aligned}
\int d \underline{\underline{x}} & \delta\left(x_{1}\right) \prod_{v \in \tau}\left(\prod_{P_{b} \in\left\{P_{b}\right\}_{v}} \prod_{i \in P_{b}}\left(\gamma^{h_{v}}\left|x_{\tau\left(v^{i}\right)}\right|\right)^{(1-\varepsilon) \delta_{n \tau_{v}^{(i)}}, 2 \delta_{Q \tau_{v}^{(i)}}, 0}\right. \\
& \left.\times \exp \left(-\widetilde{\delta} \gamma^{h_{v}} d\left(\tau_{v}\right)\right)\right) \leqq K^{n} \prod_{v \in \tau} \gamma^{-2\left(s_{v}-1\right) h_{v}} \\
& \times \prod_{P_{b} \in\left\{P_{b}\right\}_{v}} \prod_{i \in P_{b}}\left(\gamma^{-\left(h_{v}-h_{v^{\prime}}\right)}\right)^{(1-\varepsilon) \delta_{n \tau_{v}^{(i)},}, 2 \delta_{Q \tau_{v}^{(i)}}, 0} \\
= & \text { using Eq. }(8 \mathrm{~b}) \\
= & K^{n}\left(\gamma^{-2 k}\right)^{n-1} \prod_{v \in \tau} \gamma^{-\left(2 n_{v}-2\right)\left(h_{v}-h_{v^{\prime}}\right)} \\
& \times \prod_{P_{b} \in\left\{P_{b}\right\}_{v}} \prod_{i \in P_{b}}\left(\gamma^{-\left(h_{v}-h_{v^{\prime}}\right)}\right)^{(1-\varepsilon) \delta_{n \tau_{v}^{(i)}}, 2 \delta_{Q \tau_{v}^{(i)}}, 0}
\end{aligned}
$$


with $K>0$; therefore

$$
\begin{aligned}
& \left|\Gamma\left(\tau,\left\{\underline{h}_{v}\right\},\left\{\left\{P_{b}\right\}_{v}\right\},\left\{I_{v}\right\}, \underline{\underline{x}}, \underline{\underline{\sigma}}\right)\right| \leqq(K C(M, \gamma))^{n}\left(\gamma^{\left(\frac{\alpha^{2}}{4 \pi}-2\right) k}\right)^{n} \gamma^{2 k} \\
& \quad \times \prod_{v \in \tau}\left[\left(C_{3}(\gamma)\right)^{S_{v}} C_{9}^{n_{v}} \gamma^{\left(h_{v}-h_{v^{\prime}}\right)}\left[\left(\frac{\alpha^{2}}{4 \pi}-2\right) n_{v}+n_{v}+2-(1-\varepsilon) \delta_{n \tau_{v}^{(i)}}, 2 \delta_{Q \tau_{v}^{(i)}}, 0\right]\right. \\
& \quad \times \gamma^{\left.-\frac{\alpha^{2}}{4 \pi} Q_{v}^{2}\left(h_{v}-\left(h_{v^{\prime}}+1\right)\right) \chi\left(n_{v} \leqq M\right)\right] .}
\end{aligned}
$$

We have now the following result:

$$
\begin{aligned}
& \left\|a_{n q_{1}=n}^{(k)}(\Lambda, N ; \underline{\underline{x}}, \underline{\underline{\sigma}}, \underline{\theta}) \theta_{I}\right\| \leqq \sum_{\sigma_{=}} \sum_{\substack{v_{1}(\tau)=n \\
v_{2}(\tau)=0}}\left(\prod_{v \in \tau} \sum_{\left\{P_{1}\right\}_{v}} \sum_{\cup_{v} I_{v}=I}\right) \\
& \times(K C(M, \gamma))^{n}\left(\gamma^{\frac{\alpha^{2}}{4 \pi} k}\right)^{n} \gamma^{2 k} \quad \sum_{h_{0}>k} h_{v\}} \prod_{v \in \tau}\left[\left(C_{3}(\gamma)\right)^{s_{v}} C_{9}^{n_{v}}\right. \\
& \left.\times \gamma^{\left(h_{v}-h_{v^{\prime}}\right)}\left[\left(\frac{\alpha^{2}}{4 \pi}-1\right) n_{v}+2-(1-\varepsilon) \delta_{n \tau_{v}^{(i)}}, 2 \delta_{Q \tau_{v}^{(i)}}, 0\right] \gamma-\frac{\alpha^{2}}{4 \pi} Q_{v}^{2}\left(h_{v}-\left(h_{v^{\prime}}+1\right)\right) \chi\left(n_{v} \leqq M\right)\right] .
\end{aligned}
$$

Let us consider now all the $\tau$ 's with $v_{1}(\tau)>2$. It is clear that if $\alpha^{2}<2 \pi$ :

$$
\left(\left[\left(\frac{\alpha^{2}}{4 \pi}-1\right) n_{v}+2-(1-\varepsilon) \delta_{n \tau_{v}^{(i)}}, 2 \delta_{Q \tau_{v}^{(i)}}, 0\right]-\frac{\alpha^{2}}{4 \pi} Q_{v}^{2} \chi\left(n_{v} \leqq M\right)\right) \leqq-\varrho\left(\alpha^{2}\right) n_{v}
$$

with

$$
0<\frac{\alpha^{2}-2 \pi(1-\varepsilon)}{4 \pi}<\varrho\left(\alpha^{2}\right)
$$

(the worst situation is when $n_{v}=2, Q_{v}=0$ ). Therefore

$$
(9.13) \leqq\left(C_{3}(\gamma)\right)^{s_{v}} C_{9}^{n_{n}} \gamma^{-\varrho\left(\alpha^{2}\right)\left(h_{v}-h_{v^{\prime}}\right) n_{v}} \leqq \gamma^{-\left(\varrho\left(\alpha^{2}\right)-\varepsilon^{\prime}\right)\left(h_{v}-h_{v^{\prime}}\right) n_{v}},
$$

choosing $\gamma$ enough large so that

$$
\gamma^{\varepsilon^{\prime} n_{v}} \geqq\left(C_{3}(\gamma)\right)^{s_{v}} C_{9}^{n_{v}},
$$

remembering that $C_{3}(\gamma)$ depends on $\gamma$ logarithmically, it follows that:

$$
\sum_{h_{0}>k}\left\{h_{v} \prod_{v \in \tau} \gamma^{-\left(\varrho\left(\alpha^{2}\right)-\varepsilon\right)\left(h_{v}-h_{v^{\prime}}\right) n_{v}} \leqq D_{1}^{n}\right.
$$

We remember now (8.12), that $\sum_{\underline{\sigma}} \leqq(\text { const })^{n}$ and finally that

$$
\sum_{v_{1}(\tau)=n} 1=\mathscr{N} \leqq 2^{4 n}
$$

(Benfatto et al. [2]).

From all that we easily get

$$
\left\|a_{n ; q_{1}=n}^{(k)}(\Lambda, N ; \underline{\underline{x}}, \underline{\underline{\sigma}}, \underline{\theta}) \theta_{I}\right\| \leqq D_{2}^{n}\left(\gamma^{\left(\frac{\alpha^{2}}{4 \pi}-2\right) k}\right)^{n} \leqq D_{3}^{n}
$$

which is Eq. (4.7) in the case $q_{2}=0$. 
Remarks. The result we have produced is still incomplete because

a) We have not shown how to manage the case $q_{2} \neq 0$.

b) We have not considered how to treat the case of $\tau$ with $v_{1}(\tau)=2, v_{2}(\tau)=0$.

c) We have not kept explicitly trace of the volume dependence which we expect to be present in $D_{2}$ when $k=-1$.

Nevertheless once one has all the steps explicitly done for a class of trees it is easier to recognize that it is not hard to extend our result to the whole class of possible trees.

Extension to the Terms with $v_{2}(\tau)=q_{2}>0$. It is not difficult to envisage the modifications to the estimate of $(9.1)$ when $q_{2} \neq 0$.

a) $\left(\prod_{1}^{q_{1}=n} \gamma^{\frac{\alpha^{2}}{4 \pi}} h_{i}\right)$ is substituted by

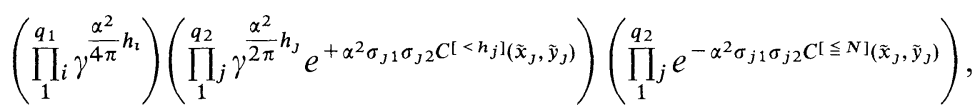

where the second factor has exactly the same origin of the first but refers to the lines of type 2. The third factor is already present in $V_{2}^{(N)}$ [see Eq. (1.1)].

$h_{j}$ is, as $h_{i}$, the frequency of the first bifurcation where the $j^{\text {th }}$ final line merge.

b) Each final line of type 2 neutral, that is with $\sigma_{j 1} \sigma_{j 2}=-1$ brings a field dependence

$$
: e^{\left.\left. \pm i \alpha\left(\sigma_{j_{1}} \varphi^{[} \leqq h_{j}\right]\left(\tilde{x}_{j}\right)-\sigma_{J_{2}} \varphi^{[} \leqq h_{j}\right]\left(\tilde{y}_{j}\right)\right)}:
$$

and this produces a "first order" zero in $\left(\tilde{x}_{j}-\tilde{y}_{j}\right)$ (the truncated expectation at level $h_{j}$ has it).

Also in the next bifurcations where only type 2 parts merge and the total charge of the bifurcation (cluster) is zero then a zero is produced when all the coordinates coincide. With these remarks the estimate $(9.7)$ has to be substituted by:

$$
\begin{aligned}
\mid \Gamma(\tau, & \left.\left\{\underline{h}_{v}\right\},\left\{\left\{P_{b}\right\}_{v}\right\},\left\{I_{v}\right\}, \underline{\underline{x}}, \underline{\underline{\sigma}}\right) \mid \\
\leqq & \left(\prod_{1}^{q_{2}} D^{[\leqq N]}\left(\tilde{x}_{j}, \tilde{y}_{j}\right) e^{-\alpha^{2} \sigma_{J 1} \sigma_{J 2}\left(C^{[} \leqq N\right]}\left(\tilde{x}_{j}, \tilde{y}_{j}\right)-C^{\left.\left[<h_{j}\right]\left(\tilde{x}_{j}, \tilde{y}_{j}\right)\right)}\right) \\
& \times\left(\prod_{1}^{q_{1}} \gamma^{\frac{\alpha^{2}}{4 \pi} h_{i}}\right)\left(\prod_{1}^{q_{2}} \gamma_{j}^{\frac{\alpha^{2}}{2 \pi} h_{j}}\right)\left(\prod_{v \in \tau} \exp -\frac{\alpha^{2}}{2}\left[U_{\varphi}^{\left[<h_{v}\right]}\left(\tau_{v}\right)-U_{\varphi}^{\left[\leq h_{v^{\prime}}\right]}\left(\tau_{v}\right)\right]\right) \\
& \times \prod_{v \in \tau}\left(\left(C_{3}(\gamma)\right)^{s_{v}} C_{10}^{n_{v}} \gamma^{\left(h_{v}-h_{v^{\prime}}\right) n_{v}^{(1)}}\right. \\
& \times \exp \left(-\delta \gamma^{h_{v}} d^{*}\left(\tau_{v}\right)\right) \prod_{P_{b} \in\left\{P_{b}\right\}_{v}} \prod_{i \in P_{b}}\left(\gamma^{h_{v}}\left|x_{\left.\tau_{v}^{(2)}\right]}\right|\right)^{(1-\varepsilon) \delta_{n_{v}}^{(1)}, 2 \delta_{n_{v}}^{(2)}, 0 \delta_{Q_{v}}, 0} \\
& \left.\times\left(\gamma^{h_{v}}\left|x_{\tau_{v}^{(2)}}\right|\right)^{(1-\varepsilon) \delta_{n_{v}}^{(1)}, 0 \delta_{n_{v}}^{(2)}, 2 \delta_{Q_{v}}, 0}\right),
\end{aligned}
$$

where in the definition of $d^{*}\left(\tau_{v}\right)$ the points $\tilde{x}_{j}$ and $\tilde{y}_{j}$ have to be thought of as connected. This point will be discussed later on. $n_{v}^{(1)}$ and $n_{v}^{(2)}$ are, respectively, the number of lines of type 1 and 2 which merge in $v$;

$$
n_{v}^{(1)}+n_{v}^{(2)}=n_{v} \text {. }
$$


The next step is, as before, to integrate over the $\underline{\underline{x}}$. At this point we use the properties of $D^{[\leqq N]}(\tilde{x}, \tilde{y})$. From the definition

$$
\begin{aligned}
D^{[\leqq N]}(\tilde{x}, \tilde{y})= & \frac{1}{L^{2}} \sum_{\substack{p \in 2 \pi / L Z^{2} \\
p_{1} \neq 0}} e^{i p(\tilde{x}-\tilde{y})} \exp \frac{-p^{2}}{\gamma^{2 N}} \exp \frac{-p_{1}^{2}}{\gamma^{2 N}} \\
= & -\frac{1}{L^{2}} \sum_{\substack{p \in 2 \pi / L Z^{2} \\
p_{1}=0}} e^{i p(\tilde{x}-\tilde{y})} \exp \frac{-p^{2}}{\gamma^{2 N}} \exp \frac{-p_{1}^{2}}{\gamma^{2 N}} \\
& +\left(\frac{1}{L^{2}} \sum_{p \in 2 \pi / L Z^{2}} e^{i p(\tilde{x}-\tilde{y})} \exp \frac{-p^{2}}{\gamma^{2 N}} \exp \frac{-p_{1}^{2}}{\gamma^{2 N}}\right) \\
= & -\frac{1}{L^{2}} \underset{\substack{p \in 2 \pi / L Z^{2} \\
p_{1}=0}}{ } e^{i p(\tilde{x}-\tilde{y})} \exp \frac{-p^{2}}{\gamma^{2 N}}+D_{c}^{[\leqq N]}(\tilde{x}, \tilde{y})
\end{aligned}
$$

and

$$
\begin{gathered}
\lim _{N \rightarrow \infty} D_{c}^{[\leqq N]}(\tilde{x}, \tilde{y})=\delta(\tilde{x}, \tilde{y}), \\
\left|D_{c}^{[\leqq N]}(\tilde{x}, \tilde{y})\right| \leqq \gamma^{2 N} e^{-\gamma^{N}|\tilde{x}-\tilde{y}|} .
\end{gathered}
$$

Then the right-hand side of (9.20) can be decomposed in $2^{q_{2}}$ terms, each one with at each $j$ or the factor $-\frac{1}{L^{2}} \sum_{\substack{p \in 2 \pi / L Z^{2} \\ p_{1}=0}} e^{i p(\tilde{x}-\tilde{y})} \exp \frac{-p^{2}}{\gamma^{2 N}}$ or the factor $D_{c}^{[\leqq N]}$.

We will consider first the two extreme cases: a) at each $j$ there is the factor $D_{c}^{[\leqq N]}(\tilde{x}, \tilde{y})$;

b) at each $j$ there is $-\frac{1}{L^{2}} \sum_{\substack{p \in 2 \pi / L Z^{2} \\ p, 1}} e^{i p(\tilde{x}-\tilde{y})} \exp \frac{-p^{2}}{\gamma^{2 N}}$.

From this examination we will infer easily the estimates for all the intermediate situations.

Remark. The absence of the $p_{1}=0$ term in the definition of $D^{[\leqq N]}$ looks useless. In fact all the results discussed here are valid with $D_{c}^{[\leqq N]}$ instead of $D^{[\leqq N]}$. In this case, nevertheless, the supersymmetry of the regularized theory is destroyed.

Case a. Substituting $D_{c}^{[\leqq N]}$ instead of $D^{[\leqq N]}$ in Eq. (9.20) and performing the same estimates which produce inequality (9.12) we get:

$$
\begin{aligned}
& \left|\Gamma\left(\tau,\left\{\underline{h}_{v}\right\},\left\{\left\{P_{b}\right\}_{v}\right\},\left\{I_{v}\right\}, \underline{\underline{x}}, \underline{\underline{\sigma}}\right)\right| \leqq(K C(M, \gamma))^{n}\left(\left(\gamma^{\left(\frac{\alpha^{2}}{4 \pi}-2\right) k}\right)^{q_{1}}\left(\gamma^{\left(\frac{\alpha^{2}}{\pi}-2\right) k}\right)^{q_{2}^{+}}\right. \\
& \left.\times\left(\gamma^{-k}\right)^{q^{\overline{2}}} \gamma^{-\frac{\alpha^{2}}{2 \pi} N q_{2}^{+}} \gamma^{\left(\frac{\alpha^{2}}{2 \pi}-1\right) N q_{\overline{2}}} \gamma^{2 k}\right) \prod_{v \in \tau}\left[\left(C_{3}(\gamma)\right)^{s_{v}} C_{10}^{n_{v}}\right] \\
& \times \prod_{v \in \tau}\left[\gamma^{\left(h_{v}-h_{v^{\prime}}\right)}\left[\left(\frac{\alpha^{2}}{4 \pi}-2\right) n_{v}^{(1)}+n_{v}^{(1)} \chi\left(n_{v}^{(1)}>1\right)+2\right]\right.
\end{aligned}
$$

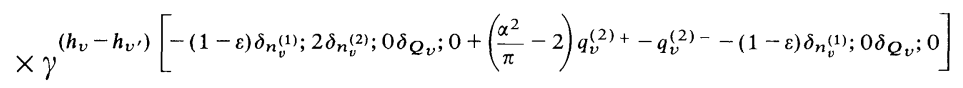

$$
\begin{aligned}
& \left.\times \gamma^{-\frac{\alpha^{2}}{4 \pi} Q_{v}^{2}\left(h_{v}-\left(h_{v}+1\right)\right) \chi\left(n_{v} \leqq M\right)}\right],
\end{aligned}
$$


where $q_{v}^{(2) \pm}=q_{2}^{ \pm} \cap n_{v} \ldots q_{2}^{+}$and $q_{2}^{-}$corresponds to those final lines associate respectively to $\sigma_{j_{1}} \sigma_{j_{2}}= \pm 1$ respectively. Of course $q_{2}^{+}+q_{2}^{-}=q_{2}$. Performing again the sum over all the frequencies, for a given tree, one immediately realizes that due to the factor $\gamma^{-\frac{\alpha^{2}}{2 \pi} N q_{2}^{+}} \gamma^{\left(\frac{\alpha^{2}}{2 \pi}-1\right) N q_{2}^{-}}$these contributions tend to zero as $N \rightarrow \infty$.

Case $b$. Here the estimate is done integrating again over all the coordinates except the $z_{j}=\left(\tilde{x}_{j}-\tilde{y}_{j}\right)$. Then the estimate becomes:

$$
\begin{aligned}
& \left|\Gamma\left(\tau,\left\{\underline{h}_{v}\right\},\left\{\left\{P_{b}\right\}_{v}\right\},\left\{I_{v}\right\}, \underline{\underline{x}}, \underline{\sigma}\right)\right| \\
& \leqq \frac{1}{|\Lambda|^{q_{2}}} \int_{\Lambda^{q_{2}}} \prod_{1}^{q_{2}}{ }_{j} d z_{j} \prod_{1}^{q_{2}} e^{-\alpha^{2} \sigma_{j 1} \sigma_{j 2}\left(C[\leqq N]\left(z_{j}\right)-C\left[<h_{j}\right]\left(z_{j}\right)\right)} \\
& \times(K C(M, \gamma))^{n}\left(\gamma^{\left(\frac{\alpha^{2}}{4 \pi}-2\right) k}\right)^{q_{1}}\left(\gamma^{\left(\frac{\alpha^{2}}{2 \pi}-2\right) k}\right)^{q_{2}} \gamma^{2 k} \prod_{v \in \tau}\left[\left(C_{3}(\gamma)\right)^{s_{v}} C_{10}^{n_{v}}\right. \\
& \times \gamma^{\left(h_{v}-h_{v^{\prime}}\right)}\left[\left(\frac{\alpha^{2}}{4 \pi}-2\right) n v^{(1)}+n v^{(1)} \chi\left(n v^{(1)}>1\right)\right] \\
& \left.\times \gamma^{\left(h_{v}-h_{v^{\prime}}\right)}\left[2-(1-\varepsilon) \delta_{n_{v}^{(1)}}^{(1)} 2 \delta_{n_{v}^{(2)}}^{(2)}, 0 \delta_{Q_{v}}, 0+\left(\frac{\alpha^{2}}{2 \pi}-2\right) n_{v}^{(2)}-(1-\varepsilon) \delta_{n_{v}^{(1)}}^{(1)} 0 \delta_{Q_{v}}, 0\right]\right] \text {. }
\end{aligned}
$$

Calling $C^{\left[h_{j} ; N\right]}=\sum_{h_{3}}^{N} C^{(h)}$ and using the decomposition

$$
e^{-\alpha^{2} \sigma_{j 1} \sigma_{j 2} C^{\left[h_{j} ; N\right]}}=\sum_{h_{j} h}^{N-1} e^{-\alpha^{2} \sigma_{j 1} \sigma_{j 2} C^{\left[h_{j} ; h\right]}}\left(e^{-\alpha^{2} \sigma_{J 1} \sigma_{J 2} C^{(h+1)}}-1\right)+e^{-\alpha^{2} \sigma_{J 1} \sigma_{J 2} C^{\left(h_{j}\right)}},
$$

one easily realizes that the first factor is bounded by $\mathscr{K}^{q_{2}}$, with $\mathscr{K}>0$. The second factor is easier to control and it gives also a term which can be bounded by $\mathscr{C}^{q_{2}}$ with $\mathscr{C}>0$. Finally it is an easy task to realize that everytime there is at least a factor $D_{c}^{[\leqq N]}$ for some $j$ the corresponding contribution is $=0$ in the limit $N \rightarrow \infty$.

Remark. The case $n=q_{2}=1$ has to be treated separately and together with the case $n=q_{1}=2$. This is done now:

The $O\left(\lambda^{2}\right)$ Contribution to $V_{\Lambda ; N}^{(k)}$. We have to discuss this contribution separately because if we try to use the previous estimates we will get a divergent result as $N \rightarrow \infty$. The $O\left(\lambda^{2}\right)$ contributions can be graphically described by the following trees:

$$
\left.V_{\Lambda ; N}^{(k)}\right|_{o\left(\lambda^{2}\right)}=\frac{}{k \quad v_{2}}+\sum_{k+1}^{N} h \frac{v_{1}}{k}
$$

Fig. 2

If we apply to the first tree the estimate (9.13) we get a divergent result as the factor $(1-\varepsilon) \delta_{n_{v}, 2} \delta_{Q_{v}, 0}$ is missing due to the lack of subsequent bifurcations after the first one at the frequency $h=h_{v}$.

Similarly the second contribution is unbounded in $N$ if $\sigma_{1} \sigma_{2}=-1$, again for the impossibility of producing a zero. In the scalar Sine-Gordon (for $\alpha^{2}>4 \pi$ ) the 
analogous problem is cured adding an appropriate constant counterterm. Here the problem is similar. The needed counterterm is, as discussed in [1]:

$$
\lambda^{2} C_{2}^{(N)}(\Lambda)=\frac{1}{2} \mathscr{E}^{T}\left(V_{2}^{(N)} ; 2\right) .
$$

It is a long but straightforward task to show that, if the theory is supersymmetric and regularized in a supersymmetric way, the following relation holds:

$$
\lambda^{2} C_{2}^{(N)}(\Lambda)=\lambda^{2} C_{2}^{(N)}(\Lambda)^{\prime}+\lambda^{2} C_{2}^{(N)}(\Lambda)^{\prime \prime}=0,
$$

where the two counterterms on the right-hand side are such that

$$
\sum_{k \neq 1}^{N} \frac{h}{k} \overbrace{v_{1}}^{v_{1}}+\lambda^{2} C_{2}^{(N)}(\Lambda)^{\prime} \text { and } \frac{v_{2}}{k}+\lambda^{2} C_{2}^{(N)}(\Lambda)^{\prime \prime}
$$

Fig. 3

have the appropriate bounds uniform in $N$ (see B. Scoppola [thesis]).

If we choose a non-supersymmetric regularization the total counterterm is not $=0$ but it is, nevertheless, finite.

The Volume Dependence. In the main result (4.7), (4.8) of this paper there is [in (4.8)] a bad volume dependence of $\left\|a_{n q_{1}}^{(-1)}(\Lambda, N ; \underline{\underline{x}}, \underline{\underline{\sigma}}, \underline{\theta}) \theta_{I}\right\|$. This is unavoidable as it is due to the fact that our theory is massless and therefore the covariances of bosons and fermions do not decay exponentially.

Here we want only to justify why in (4.7) the norm does not depend on $A$ while it does in (4.8). Intuitively it is very clear: due to the renormalization group decomposition the covariances used integrating over all the frequency fields except on the last ones $\varphi^{(0)}, \psi^{(0)}$ are all massive with a mass depending on the frequency $\sim \gamma^{h}$ if we stay on scale $h$. This is not anymore true if we integrate over the whole field.

The only technical point is the following one: in our estimates we always write

$$
e^{\frac{\alpha^{2}}{2} C[\leqq h](0)} \sim \gamma^{\frac{\alpha^{2}}{4 \pi}(h+1)}
$$

while, choosing for simplicity, the regularized covariance of Eq. (2.15), (I) we have:

$$
C^{[\leqq h]}(0)=\frac{1}{2 \pi} \log \left(\frac{\gamma^{(h+1)}}{L^{-1}}\right)=\frac{1}{2 \pi}(\log \gamma)^{(h+1)}+\frac{1}{2 \pi}(\log L) .
$$

Therefore

$$
e^{\frac{\alpha^{2}}{2} C[\leqq h](0)}=(L)^{\frac{\alpha^{2}}{4 \pi}} \gamma^{\frac{\alpha^{2}}{4 \pi}(h+1)} .
$$

The factors $(L)^{\frac{\alpha^{2}}{4 \pi}}$ have been omitted but they produce a factor $\left(L^{\frac{\alpha^{2}}{4 \pi}}\right)^{n}$, at order $n$, which has to be reinserted in the Wick product, for any $k$; the remaining volume dependences cancel between themselves if $k \geqq 0$. The same result holds with the regularized covariances which are effectively used. 


\section{Conclusions}

In this paper we proved that for $\alpha^{2}<2 \pi$ the massless supersymmetric SineGordon theory exists for weak coupling and finite volume. Moreover the theory is analytic in the sense that its Schwinger functions are analytic in $\lambda$. This result is valid both if we break the supersymmetry with the choice of the ultraviolet regularization and of the boundary conditions or if we keep the theory supersymmetric during all the regularizing steps. In this second case, as discussed in (I), we are able to compute the Witten index of the theory and to prove it is $=1$.

\section{Appendix A}

Proof of the "Factorization" Theorem. We start from Eq. (5.5) which we rewrite $(\lambda=\{i, j\})$,

$$
\begin{aligned}
& s ![(5.5)]=\sum_{g \in G_{s}^{c}} \prod_{\lambda \in g}\left[\left(e^{C(\lambda)}-1\right) e^{S(\lambda)}+\left(e^{S(\lambda)}-1\right)\right] \\
& =\sum_{g \in G_{s}^{c}}\left\{\sum_{\substack{\left.g \supset \gamma_{b}\right\} \\
\gamma_{b} \cap \gamma_{s}=\emptyset}} \prod_{b} \prod_{\lambda \in \gamma_{b}}\left(e^{C(\lambda)}-1\right) e^{S(\lambda)}\right. \\
& \times \sum_{\substack{\left.\left.g \backslash\left\{\gamma_{b}\right\}\right\} \tilde{\gamma}_{s}\right\} \\
\tilde{\gamma_{s}} \sim \tilde{\gamma}_{t}=\emptyset \\
\left[\gamma_{2}\right] \supset\left[\tilde{\gamma}_{s}\right] \text { for some } i}} \prod_{s} \prod_{\tilde{\lambda}_{\in} \in \tilde{\gamma}_{s}}\left(e^{S(\tilde{\lambda})}-1\right) \\
& \left.\times \sum_{\substack{\left.g \backslash\left\{\gamma_{b}\right\} \cup\left\{\tilde{\gamma}_{t}\right\}\right) \tilde{\gamma}_{t} \\
\left[\gamma_{t}\right] \supset\left[\tilde{\gamma}_{t}\right] ; \forall i}} \prod_{t} \prod_{\tilde{\lambda}_{\in} \in \tilde{\gamma}_{t}}\left(e^{S(\tilde{\tilde{\lambda}})}-1\right) \chi\left(\left\{\gamma_{b}\right\} \cup\left\{\tilde{\gamma}_{s}\right\} \cup\left\{\tilde{\tilde{\gamma}}_{t}\right\}=g\right)\right\} .
\end{aligned}
$$

$\left\{\gamma_{b}\right\},\left\{\tilde{\gamma}_{s}\right\},\left\{\tilde{\gamma}_{t}\right\}$ are sets of connected Mayer graphs. $[\gamma]$ is the set of vertices of the Mayer graph $\gamma \cdot \gamma$ can be thought as a set of bonds $\{\lambda\}$ sometimes denoted by $[[\gamma]]$. Therefore it is obvious the meaning of $g \supset \gamma$ or $g \backslash\left\{\gamma_{b}\right\} \supset \gamma$.

We interchange in (A.1) the order of the sums:

$$
\begin{aligned}
&(\mathrm{A} .1)=\left.\sum_{\gamma_{b} \cap \gamma_{s}=\emptyset} \hat{\gamma}_{b}\right\} \\
& \times \prod_{b} \prod_{\lambda \in \gamma_{b}}\left(e^{C(\lambda)}-1\right) \prod_{\lambda \in \gamma_{b}} e^{S(\lambda)} \\
& \times \sum_{\substack{\left.\tilde{\gamma}_{s} \cap \tilde{\gamma}_{t}\right\} \\
\left[\gamma_{i}\right] \supset\left[\tilde{\gamma}_{s}\right] \text { for some } i}} \prod_{s} \prod_{\tilde{\lambda} \in \tilde{\gamma}_{s}}\left(e^{S(\tilde{\lambda})}-1\right) \\
&\left.\times \sum_{\left[\gamma_{t}\right] \supset\left[\tilde{\gamma}_{t}\right] \text { for any } i} \prod_{t} \prod_{\tilde{\tilde{\lambda}} \in \tilde{\gamma}_{t}}\left(e^{S(\tilde{\tilde{\lambda}})}-1\right)\right) \\
&\left.\chi\left(\cup \gamma_{b} \cup \tilde{\gamma}_{t} \text { is connected }\right) \chi\left(\left[\cup \gamma_{b} \cup \tilde{\gamma}_{t}\right]\right)=\{1, \ldots, s\}\right) .
\end{aligned}
$$

The sets $\left\{\gamma_{b}\right\},\left\{\tilde{\gamma}_{s}\right\},\left\{\tilde{\gamma}_{t}\right\}$ define, with the previous conditions, a graph $g$ uniquely. We write

$$
\sum_{\gamma_{b} \cap \gamma_{s=\emptyset}}(\cdot)=\sum_{P_{b} \cap P_{s}=\emptyset} \prod_{b} \prod_{b} \sum_{\gamma_{b} \in G_{P_{b}}^{c}}(\cdot),
$$

where $\left\{P_{b}\right\}$ is a family of subsets of $\{1, \ldots, s\}\{1, \ldots, s\} \supset P_{b}, \forall b\{1, \ldots, s\} \supseteqq \cup_{b} P_{b}$.

One has to fix the obvious rules to avoid the terms in the sum which are meaningless (for instance if $\left|P_{b}\right|=1 \sum_{\gamma_{b} \in G_{P_{b}}^{c}}$ is absent). 
Similarly we have:

$$
\begin{aligned}
& \sum_{\substack{\tilde{\gamma}_{s} \cap \tilde{\gamma}_{\hat{\gamma}}=\emptyset \\
\left[\tilde{\gamma}_{i}\right] \supset\left[\tilde{\gamma}_{s}\right]}}(\cdot)=\sum_{\substack{\left.\tilde{P}_{s} \cap \tilde{P}_{t}=\emptyset \\
P_{i} \supset \tilde{P}_{s}\right\}}} \prod_{s} \sum_{\substack{\tilde{\gamma}_{s} \in G_{P_{s}}^{c} \\
\left[\tilde{P}_{s}\right.}}(\cdot)
\end{aligned}
$$

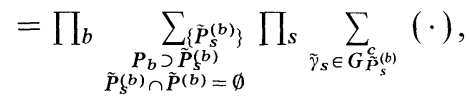

where $G_{P}^{c}$ is the set of connected Mayer graphs whose vertices $\in P$.

We rewrite (A.2) in the following way:

$$
\begin{aligned}
& \text { (A.1) }=\sum_{\boldsymbol{P}_{b} \cap \boldsymbol{P}_{s=\emptyset}\left\{\boldsymbol{P}_{b}\right\}}\left\{\prod _ { b } \left[\left(\sum_{\gamma_{b} \in G_{P_{b}}^{c}} \prod_{\lambda \in \gamma_{b}}\left(e^{C(\lambda)}-1\right)\right.\right.\right. \\
& \times \prod_{\lambda \in \gamma_{b}} e^{S(\lambda)}\left[\sum_{\substack{P_{b} \supset \tilde{P}_{b}^{(b)} \\
\tilde{P}_{s}^{(b)} \cap \tilde{P}_{t}^{(b)}=\emptyset}} \prod_{s} \sum_{\tilde{\gamma}_{s} \in G_{\tilde{P}}^{c}(b)} \prod_{\tilde{\lambda}_{s} \in \tilde{\gamma}_{s}}\left(e^{S(\tilde{\lambda})}-1\right)\right. \\
& \left.\left.\times \chi\left(\left[\left[\gamma_{b}\right]\right] \cap\left[\left[\tilde{\gamma}_{s}\right]\right]=\emptyset\right)\right]\right] \sum_{\substack{P_{1} \supset\left[\tilde{\gamma}_{t}\right\} \\
\text { for some } i}} \prod_{t} \prod_{\tilde{\tilde{\lambda}} \in \tilde{\gamma}_{t}}\left(e^{S(\tilde{\bar{\lambda}})}-1\right) \\
& \left.\times \chi\left(\cup \tilde{\gamma}_{t} \text { is connected if the } P_{i} \text { are thought of as points }\right)\right\}
\end{aligned}
$$

It is easy to recognize that

$$
\begin{aligned}
& \prod_{\lambda \in \gamma_{b}} e^{S(\lambda)}\left[\sum_{\substack{P_{b} \supset \tilde{P}^{(b)} \\
\tilde{P}(b) \cap \tilde{P}_{t}^{(b)}=\emptyset}} \prod_{s} \sum_{\tilde{\gamma}_{s} \in G_{\tilde{P}}^{c} \tilde{r}_{s}^{(b)}} \prod_{\hat{\lambda} \in \tilde{\gamma}_{s}}\left(e^{S(\tilde{\lambda})}-1\right)\right. \\
& \left.\times \chi\left(\left[\left[\gamma_{b}\right]\right] \cap\left[\left[\tilde{\gamma}_{s}\right]\right]=\emptyset\right)\right]=I_{b}=\mathscr{E}\left(\prod_{j \in P_{b}}: e^{i \psi(j)}:\right) .
\end{aligned}
$$

In fact

$$
\begin{aligned}
& \prod_{\lambda \in \gamma_{b}} e^{S(\lambda)}=\prod_{\lambda \in \gamma_{b}}\left[\left(e^{S(\lambda)}-1\right)+1\right]
\end{aligned}
$$

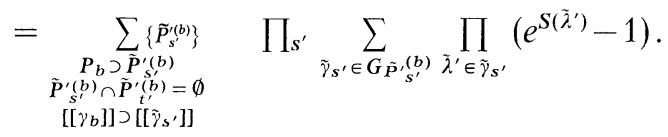

Plugging it in to (A.6) we get

$$
\begin{aligned}
& I_{b}=\sum_{\substack{P \\
P_{b} \mathcal{P}^{(p)}(b) \\
\hat{P}_{s}^{(b)} \cap \hat{\boldsymbol{P}}_{t}^{(b)}=\emptyset}} \prod_{s} \sum_{\tilde{\gamma}_{s} \in G \hat{P}_{s}^{(b)}} \prod_{\tilde{\lambda} \in \tilde{\gamma}_{s}}\left(e^{S(\tilde{\lambda})}-1\right) \\
& =\sum_{\substack{P_{b} \supset \hat{P}_{(b)}^{(b)} \\
\hat{P}_{s}^{(b)} \cap \hat{P}_{t}^{(b)}=\emptyset}} \prod_{s} \mathscr{E}^{T}\left(: e^{i \psi(j)}:\left.\right|_{\hat{P}_{s}^{(b)}}\right)=\mathscr{E}\left(\prod_{j \in P_{b}}: e^{i \psi(j)}:\right),
\end{aligned}
$$

where we used the well known relation:

$$
\mathscr{E}\left(A_{1} \ldots A_{n}\right)=\sum_{\cup_{J} Y_{J}=\{1 \ldots n\}} \prod, \mathscr{E}^{T}\left(\left.A\right|_{Y_{j}}\right)
$$

and the convention $\mathscr{E}^{T}\left(: e^{i \varphi}:\left.\right|_{\hat{P}_{s}}\right)=1$ if $\hat{P}_{s}$ is made by only a point. 
We can write also:

$$
I_{b}=\mathscr{E}\left(e^{i \Sigma_{j \in P_{b}} \psi(j)}\right)\left(\prod_{j \in P_{b}} \mathscr{E}\left(e^{i \psi(j)}\right)\right)^{\cdot 1} .
$$

Equation (A.5) becomes:

$$
\begin{aligned}
& \text { (A.1) }=\sum_{P_{b} \cap P_{s=\emptyset}\left\{P_{b}\right\}}\left\{\left(\prod_{b} I_{b}\right) \prod_{b} \mathscr{E}^{T}\left(: e^{i \varphi}:\left.\right|_{P_{b}}\right)\right. \\
& \times\left[\sum_{\substack{P_{t} \ngtr\left[\tilde{\gamma}_{t}\right\} \\
\text { for some } i}} \prod_{t} \prod_{\tilde{\tilde{\lambda}} \in \tilde{\gamma}_{t}}\left(w^{S(\tilde{\tilde{\lambda}})}-1\right)\right. \\
& \left.\left.\times \chi\left(\cup \tilde{\gamma}_{t} \text { is connected if the } P_{b} \text { are thought of as points }\right)\right]\right\} \text {. }
\end{aligned}
$$

We now look at the [ ] part of (A.11). Equation (A.11) is just the sum over all the possible Mayer graphs (connected) between the following $t+\tilde{s}$ vertices:

$P_{1}, \ldots, P_{t}$ and the remaining $\{1, \ldots, s\} \backslash \bigcup_{1}^{t} P_{b}$ vertices.

$$
\left(\tilde{S}=s-\sum_{i}^{t}\left|P_{i}\right|=s-|\mathscr{P}|\right) .
$$

To each of these vertices is associated $: e^{i \psi(V)}:$, where

$$
\psi\left(P_{b}\right)=\sum_{j \in P_{b}} \psi(j)
$$

Therefore

$$
(\mathrm{A} .11)=\mathscr{E}^{T}\left(: e^{i \psi\left(P_{1}\right)}:, \ldots,: e^{i \psi\left(P_{t}\right)}:,: e^{i \psi\left(j_{1}\right)}:, \ldots,: e^{i \psi\left(j_{5}\right)}:\right)
$$

and

$$
\prod_{b} I_{b}(\mathrm{~A} .11)=\mathscr{E}^{T}\left(e^{i \psi\left(\boldsymbol{P}_{1}\right)}, \ldots, e^{i \psi\left(\boldsymbol{P}_{t}\right)}, e^{i \psi\left(j_{1}\right)}, \ldots, e^{i \psi\left(j_{s}\right)}\right) \prod_{1}^{s} i \mathscr{E}\left(e^{-i \psi(i)}\right)
$$

\section{Appendix B}

We collect the estimates needed to prove the inequality (9.7); more details can be found in Gallavotti and Nicolò [9] and Benfatto et al. [2],

$$
U_{\varphi}^{()}(\tau)=\left\langle\left(\sum_{i \in \tau} \sigma_{i} \varphi^{()}\left(x_{i}\right)\right)^{2}\right\rangle \geqq 0
$$

Therefore

$$
\begin{aligned}
& U_{\varphi}^{\left[<h_{v}\right]}\left(\tau_{v}\right)-U_{\varphi}^{\left[<h_{v^{\prime}}\right]}\left(\tau_{v}\right)=\sum_{i ; j \in \tau_{v}} \sigma_{i} \sigma_{j}\left(C^{\left[<h_{v}\right]}\left(x_{i}, x_{j}\right)-C^{\left[<h_{v^{\prime}}\right]}\left(x_{i}, x_{j}\right)\right) \\
& \quad=\sum_{h_{v^{\prime}+1} h}^{h_{v}-1}\left[\sum_{i ; j \in \tau_{v}} \sigma_{i} \sigma_{j}\left[\left(C^{(h)}\left(x_{i}, x_{j}\right)-C^{(h)}(0)\right)+C^{(h)}(0)\right]\right] \\
& \leqq\left(h_{v}-\left(h_{v^{\prime}}+1\right)\right) C^{(h)}(0) Q_{v}^{2}+K_{1} n_{v}^{2} \gamma\left(\gamma^{h_{v}} d\left(\tau_{v}\right)\right)^{1-\varepsilon},
\end{aligned}
$$

where $K_{1}$ is a constant $>0, \varepsilon>0$, and $d\left(\tau_{v}\right)$ is the length of the shortest path connecting all the points of $\tau_{v}$. 
We used the easy estimate

$$
\left|C^{(h)}\left(x_{i}, x_{j}\right)-C^{(h)}(0)\right| \leqq K_{1} \gamma\left(\gamma^{h}\left|x_{i}-x_{j}\right|\right)^{1-\varepsilon},
$$

then we use the following estimate:

$$
\begin{aligned}
& \exp \left(-\frac{\alpha^{2}}{2}\left[U_{\varphi}^{\left[<h_{v}\right]}\left(\tau_{v}\right)-U_{\varphi}^{\left[<h_{v^{\prime}}\right]}\left(\tau_{v}\right)\right] \leqq 1\right), \quad \text { if } n_{v} \geqq M \\
& \exp \left(-\frac{\alpha^{2}}{2}\left[U_{\varphi}^{\left[<h_{v}\right]}\left(\tau_{v}\right)-U_{\varphi}^{\left[<h_{v^{\prime}}\right]}\left(\tau_{v}\right)\right]\right) \\
& \leqq \gamma^{-\frac{\alpha^{2}}{4 \pi} Q_{v}^{2}\left(h_{v}-\left(h_{v^{\prime}}+1\right)\right)} \exp \left(K_{1} M^{2} \gamma\left(\gamma^{h_{v}} d\left(\tau_{v}\right)\right)^{1-\varepsilon}\right), \text { if } n_{v}<M
\end{aligned}
$$

and $M$ will be appropriately chosen.

In Gallavotti and Nicolò [9] the following inequality is proven:

$$
\sum_{v \in \tau} \gamma^{h_{v}} d^{*}\left(\tau_{v}\right) \geqq\left(1-\gamma^{-1}\right) \sum_{v \in \tau} \gamma^{h_{v}} d\left(\tau_{v}\right) .
$$

Therefore

$$
\begin{aligned}
& \prod_{v \in \tau} \exp \left(\left(K_{1} M^{2} \gamma\left(\gamma^{h_{v}} d\left(\tau_{v}\right)\right)^{1-\varepsilon} \chi\left(n_{v}<M\right)\right)-\gamma \gamma^{h_{v}} d^{*}\left(\tau_{v}\right)\right) \\
& \leqq {\left[\prod_{v \in \tau} \exp \left(\left(K_{1} M^{2} \gamma\left(\gamma^{h_{v}} d\left(\tau_{v}\right)\right)^{1-\varepsilon} \chi\left(n_{v}<M\right)\right)-\frac{\gamma}{2}\left(1-\gamma^{-1}\right) \gamma^{h_{v}} d\left(\tau_{v}\right)\right)\right] } \\
& \gamma^{-\frac{\alpha^{2}}{4 \pi} Q_{v}^{2}\left(h_{v}-\left(h_{v^{\prime}}+1\right)\right)} \prod_{v \in \tau} \exp \left(\tilde{\gamma} \gamma^{h_{v}} d\left(\tau_{v}\right)\right) \leqq(C(M, \gamma))^{n} \prod_{v \in \tau} \exp \left(\tilde{\gamma} \gamma^{h_{v}} d\left(\tau_{v}\right)\right) \\
&\left(\tilde{\gamma}=\frac{\gamma}{2}\left(1-\gamma^{-1}\right)\right) .
\end{aligned}
$$

The following equations will be used next:

$$
\begin{gathered}
\sum_{1}^{n} h_{i}=\sum_{v \geqq v_{0}} n_{v}\left(h_{v}-h_{v^{\prime}}\right)+n k, \\
\sum_{v \geqq v_{0}}\left(s_{v}-1\right) h_{v}=\sum_{v \geqq v_{0}}\left(h_{v}-h_{v^{\prime}}\right)\left(n_{v}-1\right)+k(n-1)
\end{gathered}
$$

( $k$ is the "root" of the tree). Their proof is trivial (see for instance Benfatto et al. [2]). Therefore

$$
\prod_{1}^{n} \gamma^{\frac{\alpha^{2}}{4 \pi} h_{t}}=\left(\prod_{v \in \tau} \gamma^{\frac{\alpha^{2}}{4 \pi} n_{v}\left(h_{v}-h_{v^{\prime}}\right)}\right)\left(\gamma^{\frac{\alpha^{2}}{4 \pi} k}\right)^{n} .
$$

We will use also the following expression:

$$
\prod_{v \in \tau} \gamma^{-2\left(s_{v}-1\right) h_{v}}=\left(\gamma^{-2 k}\right)^{n-1} \prod_{v \in \tau} \gamma^{\left(-2 n_{v}+2\right)\left(h_{v}-h_{v^{\prime}}\right)}
$$

which follows from (B.8). 


\section{References}

1. Gallavotti, G.: Renormalization theory and ultraviolet stability for scalar fields via renormalization group methods. Rev. Mod. Phys. 57, 2 (1985)

Gallavotti, G., Nicolò, F.: Renormalization theory in four dimensional scalar fields (I). Commun. Math. Phys. 100, 545 (1985), and Renormalization theory in four dimensional scalar fields (II). Commun. Math. Phys. 101, 247 (1985)

2. Benfatto, G., Gallavotti, G., Nicolo, F.: The dipole phase in the two dimensional Coulomb gas. Commun. Math. Phys. 106, 277 (1986)

Benfatto, G., Gallavotti, G., Nicolò, F.: On the analyticity of the pressure in the hierarchical dipole gas. (Submitted to J. Stat. Phys.)

3. Pordt, A.: Mayer expansion for euclidean lattice field theory: Convergence properties and relation with perturbation theory, Preprint ISSN 0418-9833 (1985) Institut für Theoretische Physik, Universität Hamburg

4. Battle, G., Federbush, P.: A phase cell cluster expansion for Euclidean field theories. Ann. Phys. 142, 95 (1982)

5. Glimm, J., Jaffe, A., Spencer, T.: The particle structure of the weakly-coupled $P(\Phi)_{2}$ model and other applications. High-temperature expansion. In: Constructive quantum field theory. Velo, G., Wightman, A.S. (eds.). Berlin, Heidelberg, New York: Springer 1973

6. Gopfert, M., Mack, G.: Iterated Mayer expansion for classical gases at low temperatures. Commun. Math. Phys. 81, 97 (1981)

7. Gawedzki, K., Kupiainen, A.: Gross-Neveu model through convergent perturbation expansions. Commun. Math. Phys. 102, 1 (1985)

8. Gallavotti, G.: Renormalization theory and ultraviolet stability for scalar fields via renormalization group methods. Rev. Mod. Phys. 57, 2 (1985)

9. Gallavotti, G., Nicolò, F.: The "screening Phase Transitions" in the two dimensional Coulomb gas. J. Stat. Phys. 39, 133 (1985)

Communicated by K. Gawedzki

Received September 13, 1988 